تأثير المجموعة الوراثية ومصدر دهن العليقة في الأداء الإنتاجي لأمهات طائر السلوى

\author{
ماجد أحمد صبري النعيمي

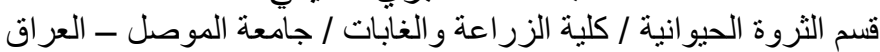 \\ E-mail: majid_aln@yahoo.com
}

\begin{abstract}
الخلاصة

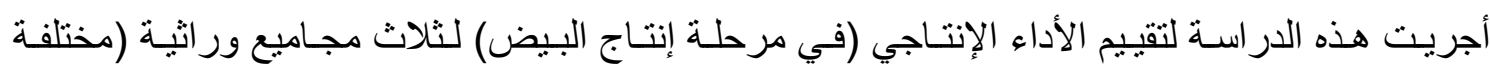

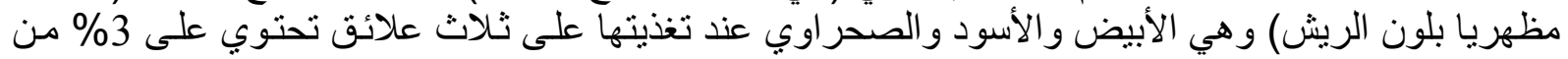

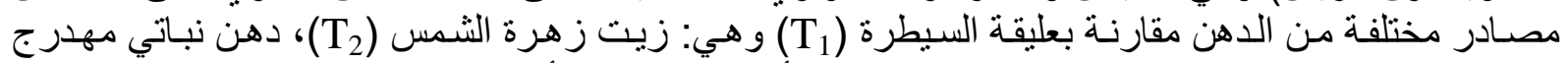
وشتحم حيو اني (T)
\end{abstract}

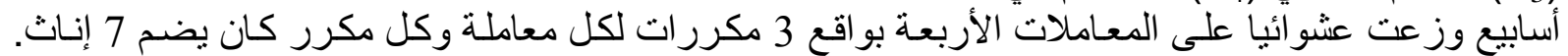

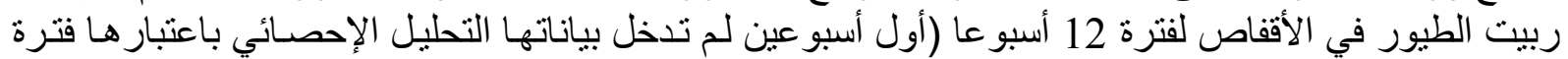

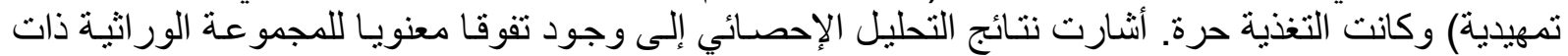

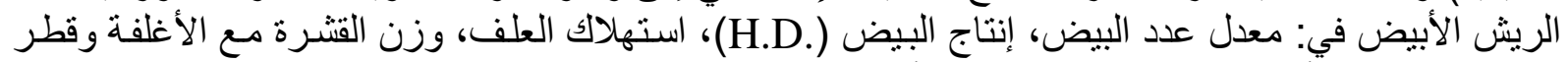

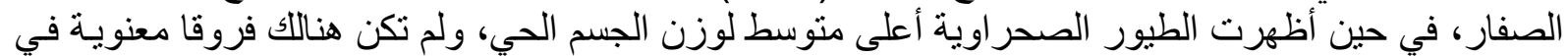

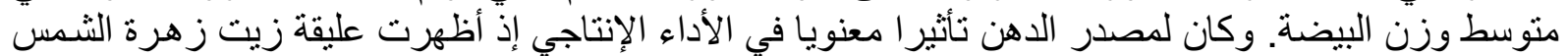

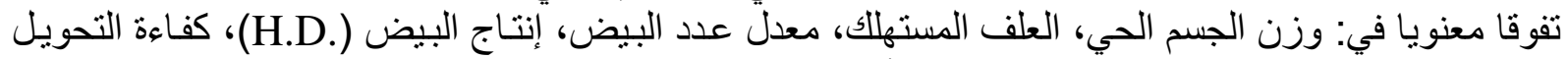

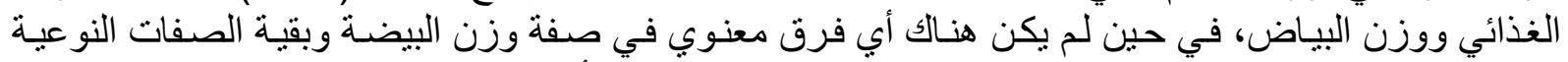

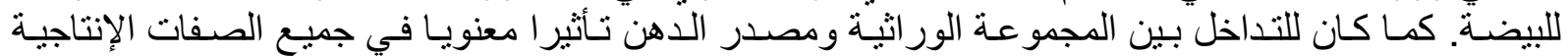

باستثناء وزن البيضة.

الكلمات الدالة: المجموعة اليضة: الور اثية، مصدر الدهن، طائر السلوى (مرحلة إنتاج البيض).

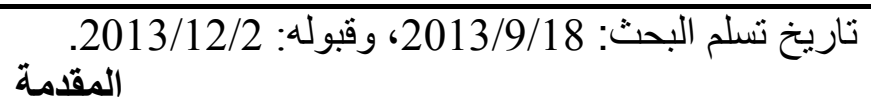

لقد أصبح طائر السلوى في الوقت الحاضر من الطيور الثـائعة التربية في العديد من دوبة دول العـالم والدول

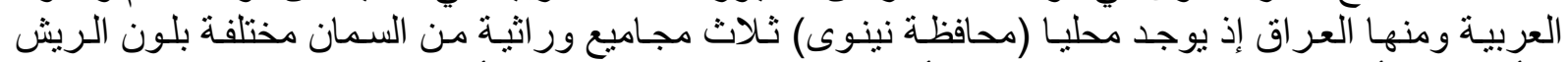

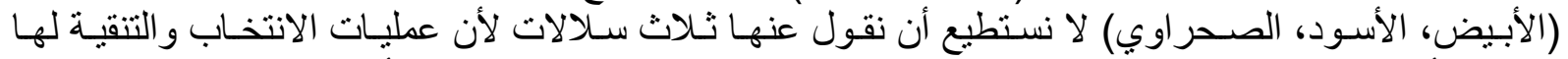

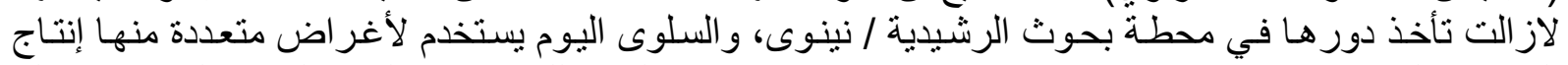

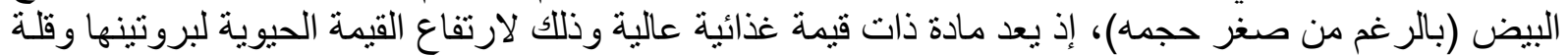

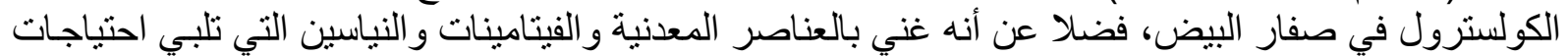

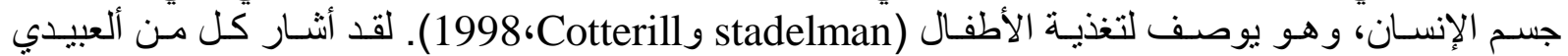

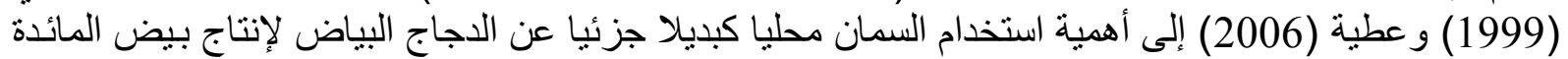

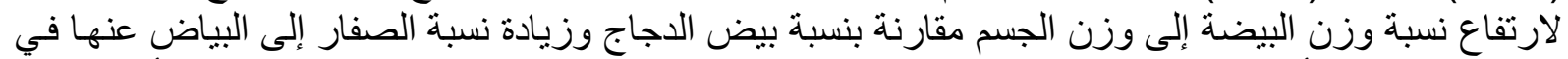

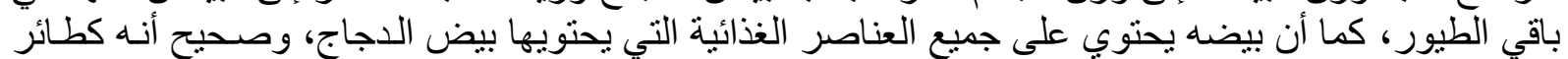

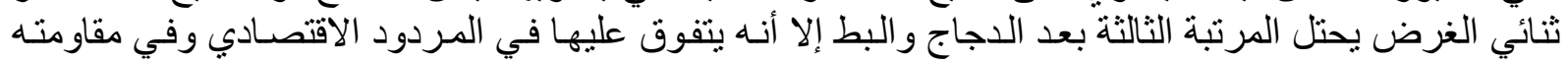

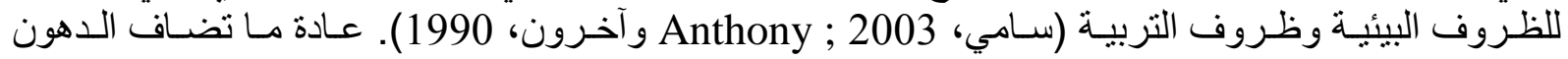

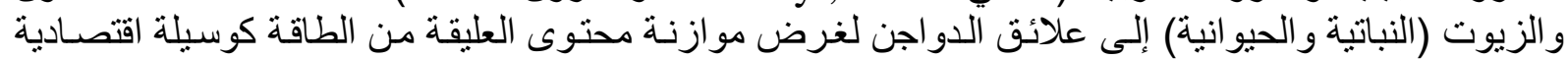

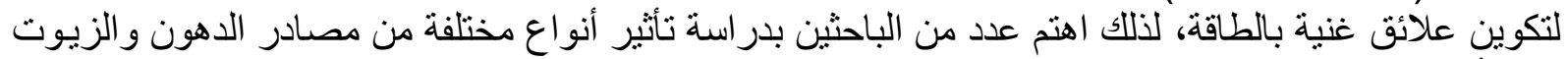

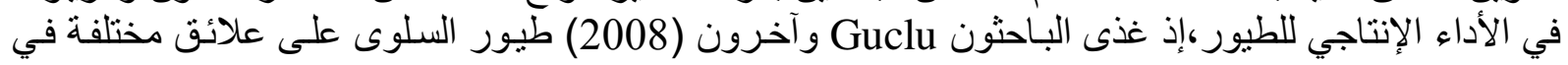

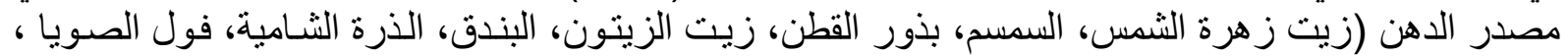

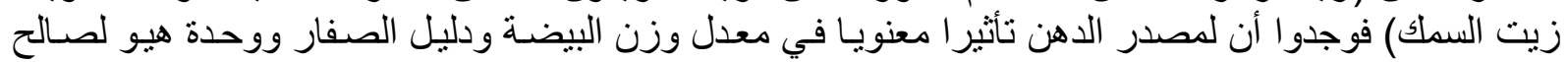

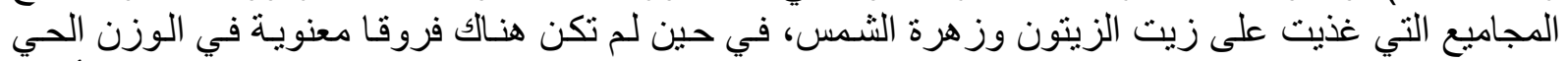

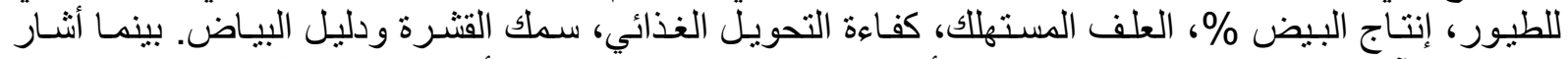

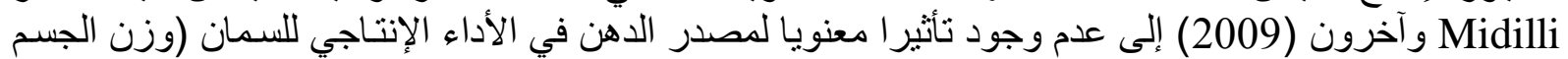

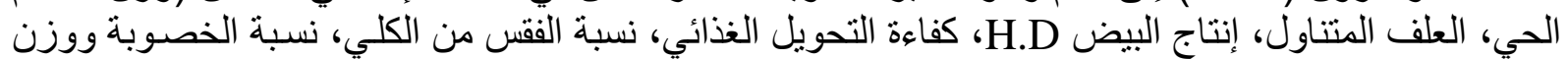

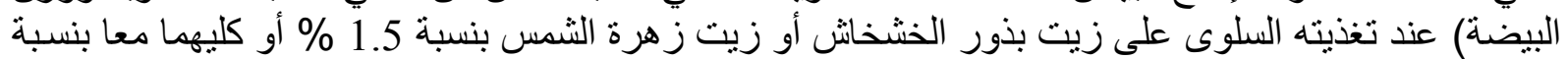

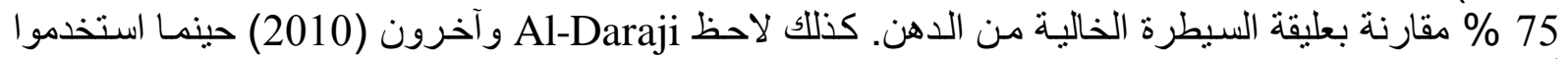

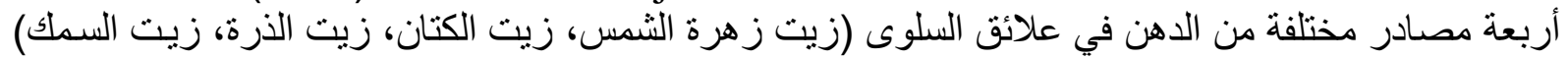


بنسبة 3 \% في مرحلـة إنتاج البيض أن الطيور المغذاة على عليقة زيت السـك أعطت أفضـل أداء إنتاجي

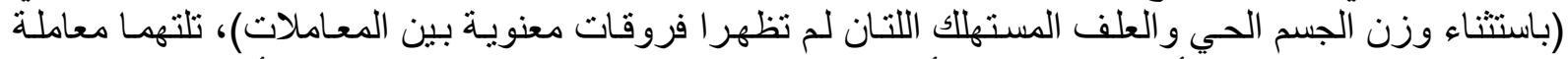

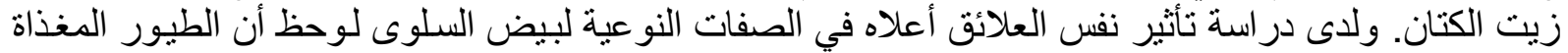

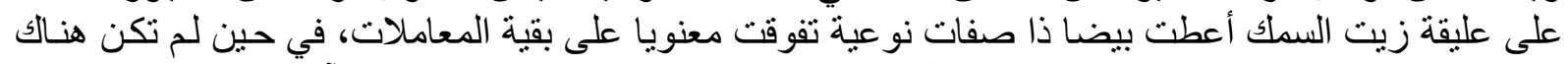

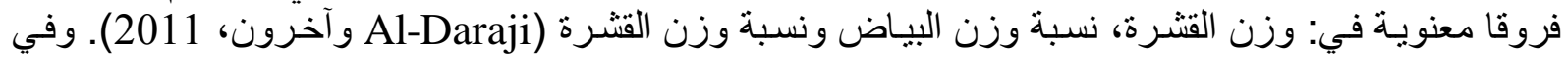

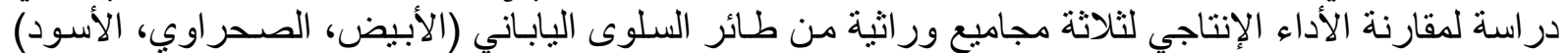

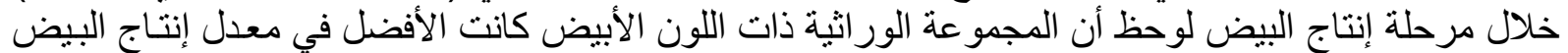
\% H.H

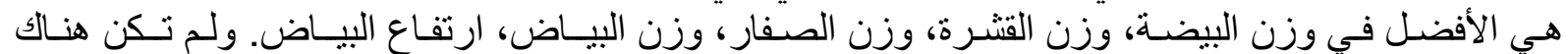

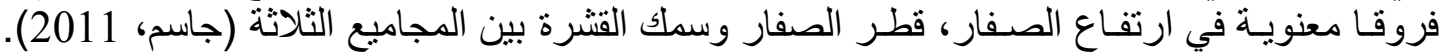

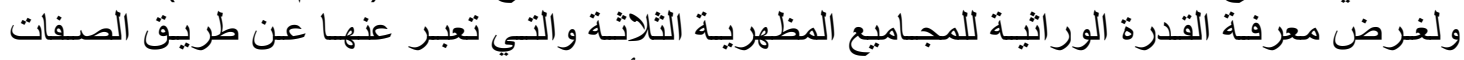

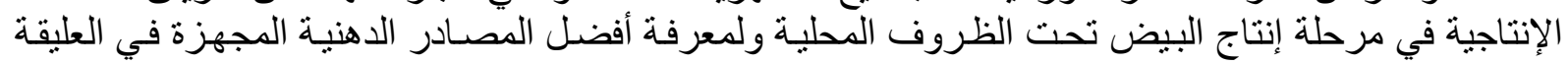

\section{مواد البحث وطر ائقه}

صممت هذه الدراسة.

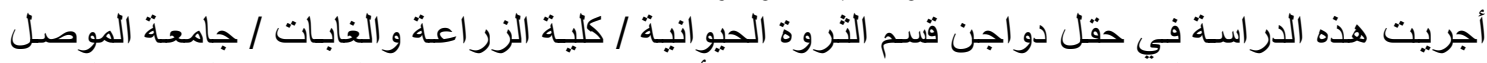

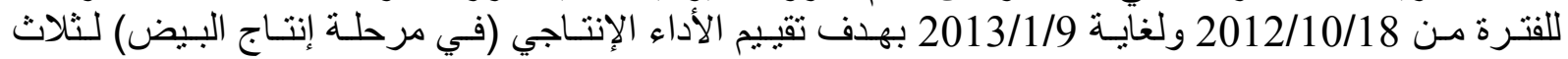

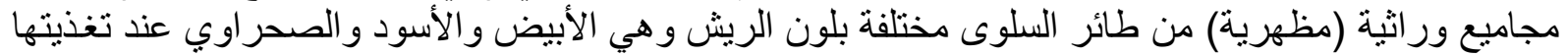

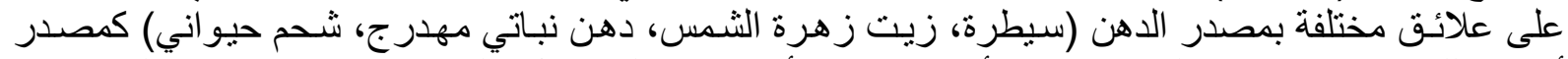

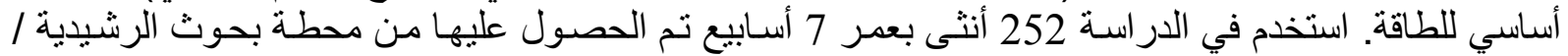

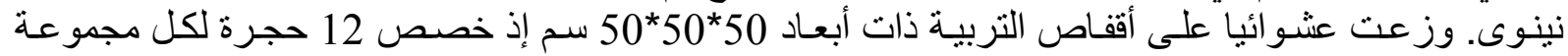

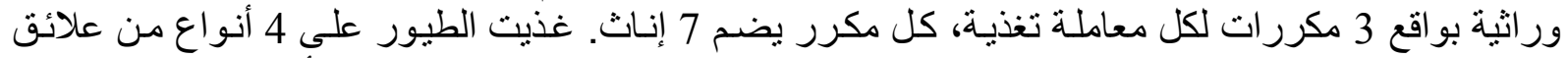

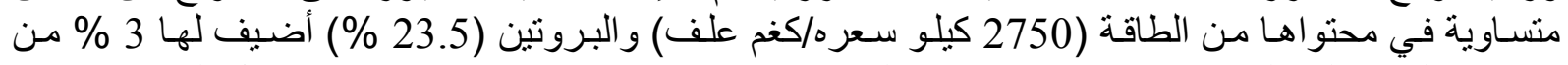

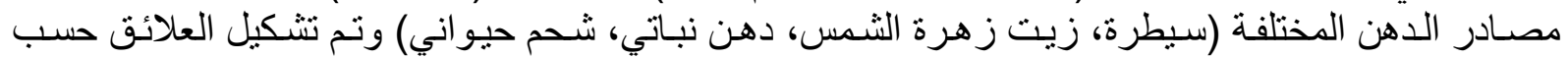
(1994،Anonymous) أسبو عيا بشكل جماعي لكل مكرر ووزن العلف المتبقي أسبو عيا وتم طرحسه من العلف المقدم لحسـاب كميات

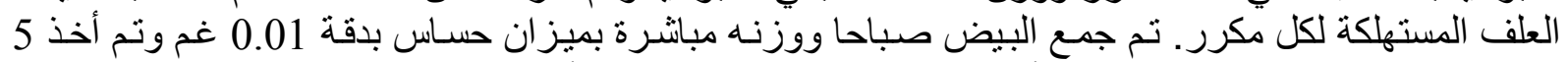

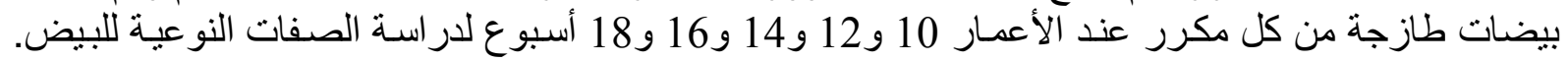

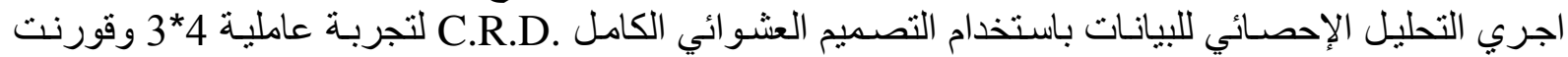

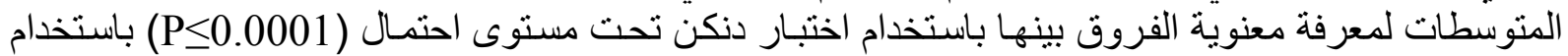

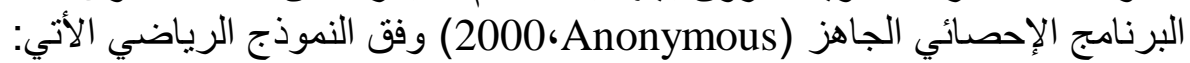
$\mathrm{Y}_{\mathrm{ijk}}=\mu+\mathrm{G}_{\mathrm{i}}+\mathrm{F}_{\mathrm{j}}+(\mathrm{GF})_{\mathrm{ij}}+\mathrm{e}_{\mathrm{ijk}}$

اذ أن: ال = قيمة أي مشاهده في التجربة. = = المتوسط التعام للمشاهدات.

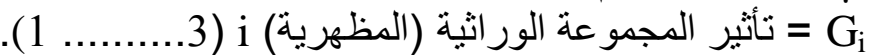

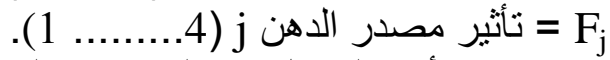

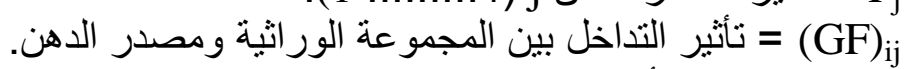

\section{النتائج والمناقشة} = e e

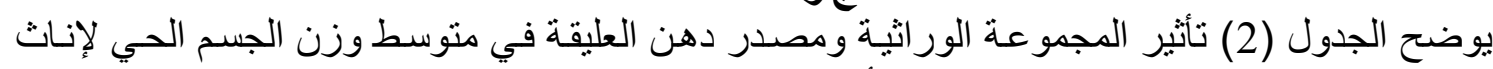

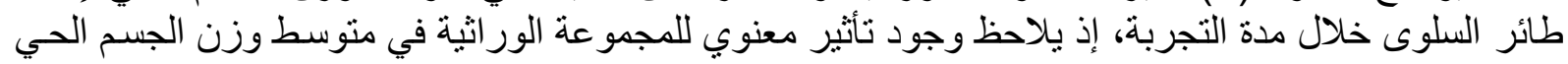

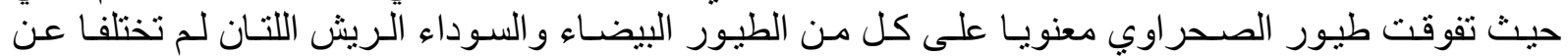

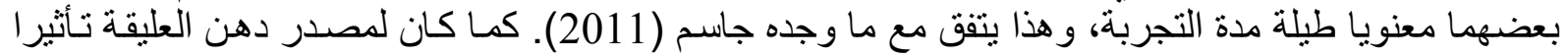

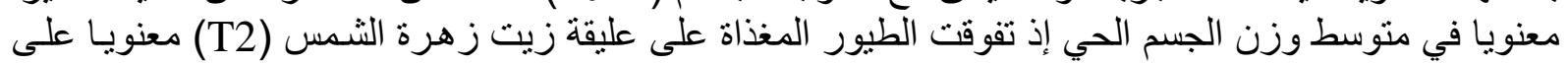

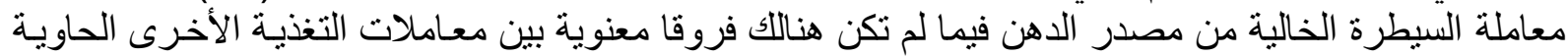

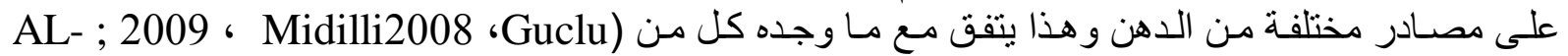
Daraji 
Mesopotamia J. of Agric.

Vol. (45) No. (4) 2017
ISSN: 2224 - 9796 (Online)

ISSN: 1815 - $316 \mathrm{X}$ (Print)

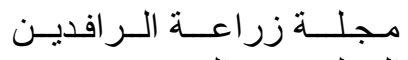

المجلد (45) العدد (4) 2017

الجدول (1): النسب المئوية لمكونات العلائق المستخدمة في التجربة

Table (1): Composition of experiment diets.

\begin{tabular}{|c|c|c|c|c|}
\hline $\mathrm{T} 4$ & $\mathrm{~T} 3$ & $\mathrm{~T} 2$ & $\mathrm{~T} 1$ & $\begin{array}{c}\text { المواد العلفية } \\
\text { Feed Stuffs }\end{array}$ \\
\hline 43.37 & 40.29 & 44.65 & 51.46 & ذرة صفر اء Yellow Corn \\
\hline 44.51 & 38.04 & 44.01 & 24.36 & $\begin{array}{l}\text { كسبة فول الصويا } 48 \text { \% بروتين Protein } \\
\text { Soybean Meal 48\% }\end{array}$ \\
\hline 1.45 & 6.46 & -------- & ----- & Wheat bran نخالة \\
\hline -------- & 5.80 & 0.85 & 21.02 & $\begin{array}{c}\text { * مركز بروتيني } \\
\text { Protein Concentrate }\end{array}$ \\
\hline 4.27 & 3.73 & 4.19 & 2.3 & حجر الكلس Limestone \\
\hline 2.9 & 2.18 & 2.8 & 0.36 & $\begin{array}{c}\text { فوسفات ثنائي الكالسيوم } \\
\text { Dicalcium Phosphate }\end{array}$ \\
\hline 0.25 & 0.25 & 0.25 & 0.25 & Salt ملح طعام \\
\hline 0.25 & 0.25 & 0.25 & 0.25 & فيتامينات ومعادن Premix \\
\hline 3 & 3 & 3 & ------ & Fat دهن \\
\hline 100 & 100 & 100 & 100 & المجموع ال Total \\
\hline \multicolumn{5}{|c|}{ التحليل الكيميائي المحسوب } \\
\hline 2750 & 2750 & 2750 & 2750 & $\begin{array}{l}\text { طاقة ممثلة كيلوسعرة/كغم علف keabolic Energy kcal/kg } \\
\text { Metabolic }\end{array}$ \\
\hline 23.5 & 23.5 & 23.5 & 23.5 & Crude Protein \% بروتين خام \\
\hline 1.32 & 1.39 & 1.33 & 1.60 & Lysine لايسين \\
\hline 0.73 & 0.90 & 0.76 & 1.34 & $\begin{array}{c}\text { مثيونين + سستين } \\
\text { Methionine+ Cystine }\end{array}$ \\
\hline 2.4 & 2.4 & 2.4 & 2.4 & Calcium \\
\hline 0.7 & 0.7 & 0.7 & 0.7 & Phosphor \\
\hline 4.23 & 4.38 & 4.08 & 3.25 & ألياف خام Crude Fiber \\
\hline 4.95 & 5.28 & 5.06 & 3.20 & eather extract مستخلص أيثز \\
\hline 117.02 & 117.02 & 117.02 & 117.02 & $\begin{array}{c}\text { نسبة الطاقة: البروتين } \\
\text { C/P ratio }\end{array}$ \\
\hline
\end{tabular}

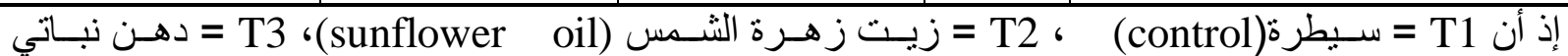

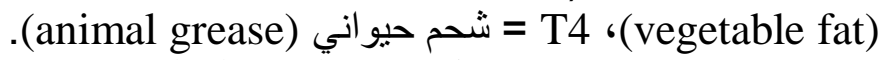

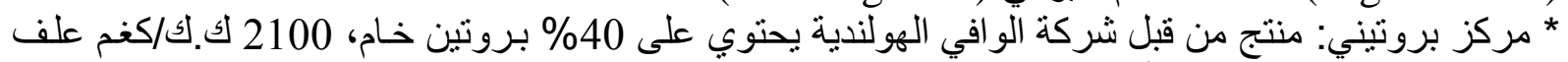

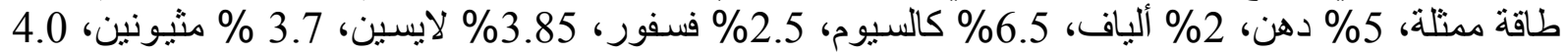
\% مثيو نين+ سنتين

أما بالنسب للتداخل بين المجموعة الور اثيـة ومصدر دهن العليقة فقد كانت الطيور الصحر اوية المغذاة

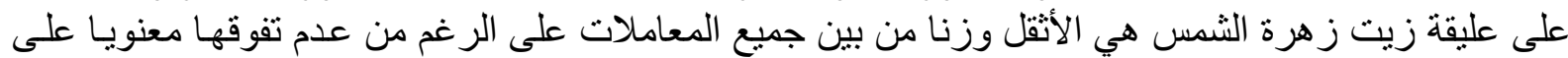

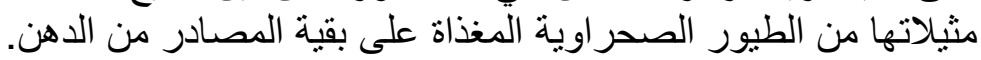

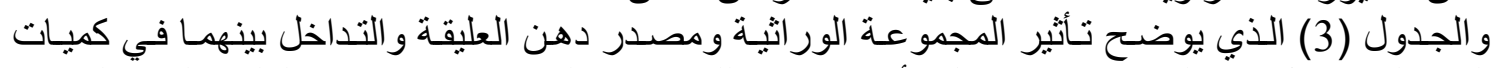

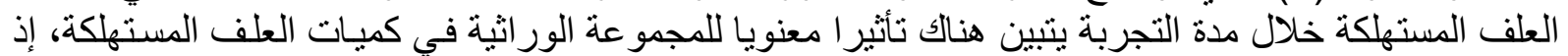

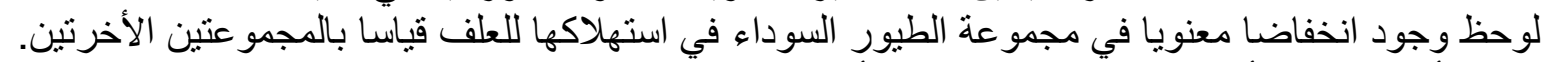

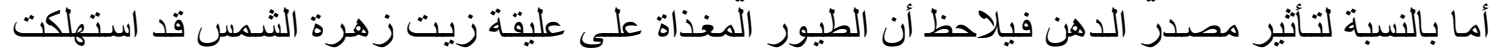

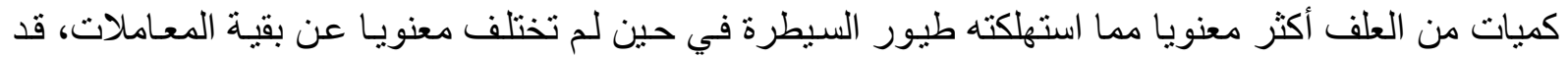

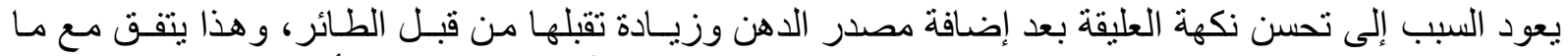

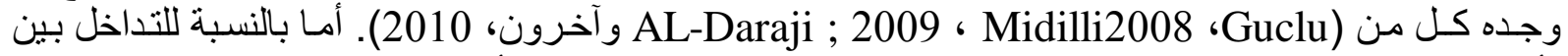

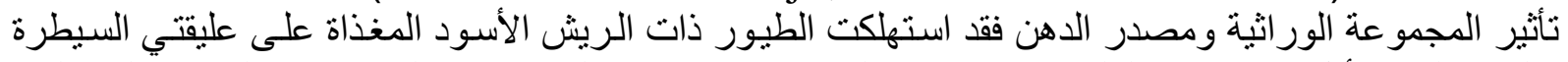

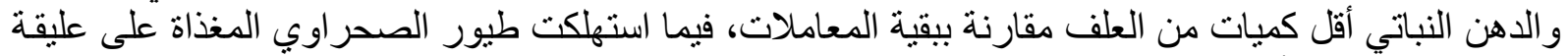
زيت زهرة الثمس أعلى الكميات. 
الجدول (2): تأثثر المجموعة الوراثية ومصدر دهن العليقة والتداخل بينهما في منوسط وزن الجسم الحي (غم).

Table (2): Effect of genetic group , ratio oil source and their interaction on live body weight $(\mathrm{g})$.

\begin{tabular}{|c|c|c|c|c|c|c|}
\hline \multicolumn{5}{|c|}{ Periods الفترات } & \multirow{2}{*}{\multicolumn{2}{|c|}{ Treatment المعاملة }} \\
\hline 5 & 4 & 3 & 2 & 1 & & \\
\hline & & $\overline{\text { Genetic grou }}$ & عة الور اثية fect & تأتثِر 1 & & \\
\hline $212.85 \mathrm{~b}$ & $212.05 \mathrm{ab}$ & $209.88 \mathrm{~b}$ & $212.88 \mathrm{~b}$ & $219.45 \mathrm{~b}$ & \multicolumn{2}{|c|}{ الأبيض White } \\
\hline $208.97 \mathrm{~b}$ & $203.82 \mathrm{~b}$ & $197.92 \mathrm{~b}$ & $208.86 \mathrm{~b}$ & $213.88 \mathrm{~b}$ & \multicolumn{2}{|c|}{ الأسود Black } \\
\hline $230.04 \mathrm{a}$ & $224.02 \mathrm{a}$ & $225.56 \mathrm{a}$ & $233.54 \mathrm{a}$ & $239.00 \mathrm{a}$ & \multicolumn{2}{|c|}{ الصحراوي Desert } \\
\hline \multicolumn{7}{|c|}{ Ratio oil source effect تأتثير مصدر دهن العليقة } \\
\hline $209.43 \mathrm{~b}$ & $204.36 \mathrm{~b}$ & $201.88 \mathrm{~b}$ & $206.85 \mathrm{~b}$ & $211.39 \mathrm{~b}$ & \multicolumn{2}{|c|}{ سيطرة T1 } \\
\hline $228.82 \mathrm{a}$ & $226.60 \mathrm{a}$ & $220.76 \mathrm{a}$ & $228.73 \mathrm{a}$ & $231.85 \mathrm{a}$ & \multicolumn{2}{|c|}{ زيت زهرة الثمس T2 } \\
\hline $214.33 \mathrm{ab}$ & $209.89 \mathrm{ab}$ & $209.90 \mathrm{ab}$ & $216.14 \mathrm{ab}$ & $227.26 \mathrm{ab}$ & \multicolumn{2}{|c|}{ T3 دهن نباتي T3 } \\
\hline $216.57 \mathrm{ab}$ & $212.33 \mathrm{ab}$ & $211.94 \mathrm{ab}$ & $221.98 \mathrm{ab}$ & $225.93 \mathrm{ab}$ & \multicolumn{2}{|c|}{ شحم حيو اني T4 } \\
\hline \multicolumn{7}{|c|}{ Interaction effect (genetic group x ratio oil source)(مصدر دهن العليقة) X نأثير التداخلَ (المجموعة الور اثئية } \\
\hline $200.63 \mathrm{~b}$ & $194.38 \mathrm{bc}$ & $193.75 \mathrm{c}$ & $194.71 \mathrm{~cd}$ & $193.33 \mathrm{c}$ & $\mathrm{T} 1$ & \multirow{4}{*}{$\begin{array}{l}\text { الأبيض } \\
\text { White }\end{array}$} \\
\hline $216.95 \mathrm{ab}$ & $222.29 \mathrm{ab}$ & $224.24 \mathrm{ab}$ & $226.64 \mathrm{abc}$ & $229.56 \mathrm{ab}$ & $\mathrm{T} 2$ & \\
\hline $224.17 \mathrm{ab}$ & $224.31 \mathrm{ab}$ & $214.79 \mathrm{abc}$ & 222.92 abcd & $241.22 \mathrm{ab}$ & T3 & \\
\hline $209.67 \mathrm{~b}$ & $207.20 \mathrm{abc}$ & $206.72 \mathrm{bc}$ & 207.24 abcd & $213.67 \mathrm{bc}$ & $\mathrm{T} 4$ & \\
\hline $202.14 \mathrm{~b}$ & $198.34 \mathrm{bc}$ & $194.88 \mathrm{c}$ & $203.34 \mathrm{bcd}$ & $212.97 \mathrm{bc}$ & $\mathrm{T} 1$ & \multirow{4}{*}{$\begin{array}{l}\text { الأسود } \\
\text { Black }\end{array}$} \\
\hline $220.98 \mathrm{ab}$ & $218.55 a b c$ & $202.82 \mathrm{bc}$ & $218.38 \mathrm{abcd}$ & $227.92 \mathrm{ab}$ & $\mathrm{T} 2$ & \\
\hline $199.93 \mathrm{~b}$ & $186.97 \mathrm{c}$ & $190.18 \mathrm{c}$ & $191.58 \mathrm{~d}$ & $192.50 \mathrm{c}$ & T3 & \\
\hline $212.84 \mathrm{~b}$ & $211.42 a b c$ & $203.81 \mathrm{bc}$ & $222.16 \mathrm{abcd}$ & 222.12abc & $\mathrm{T} 4$ & \\
\hline $225.53 \mathrm{ab}$ & $220.38 \mathrm{ab}$ & 216.99abc & $222.50 \mathrm{abcd}$ & $227.86 \mathrm{ab}$ & $\mathrm{T} 1$ & \multirow{4}{*}{ الصحراوي } \\
\hline $248.53 \mathrm{a}$ & $238.94 \mathrm{a}$ & $235.22 \mathrm{a}$ & $241.18 \mathrm{a}$ & $238.08 \mathrm{ab}$ & $\mathrm{T} 2$ & \\
\hline $218.89 \mathrm{ab}$ & $218.41 \mathrm{abc}$ & $224.73 \mathrm{ab}$ & $233.93 \mathrm{ab}$ & $248.06 \mathrm{a}$ & $\mathrm{T} 3$ & \\
\hline $227.20 \mathrm{ab}$ & $218.36 \mathrm{abc}$ & $225.29 \mathrm{ab}$ & $236.54 \mathrm{ab}$ & $242.00 \mathrm{ab}$ & $\mathrm{T} 4$ & \\
\hline
\end{tabular}

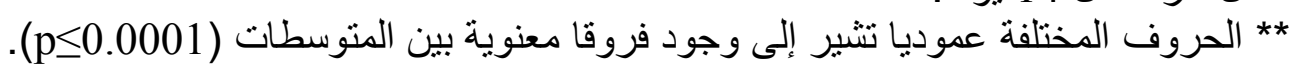

ويوضح الجدول (4) تأثثر المجموعة الور اثية ومصدر دهن العليقة والتداخل بينهما في عدد البيض المنتج

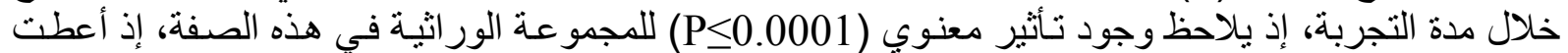

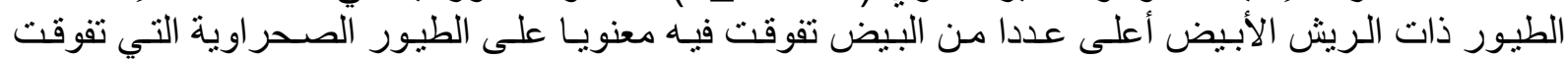

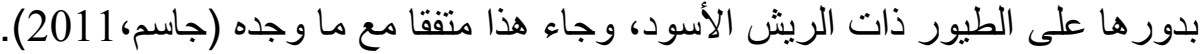

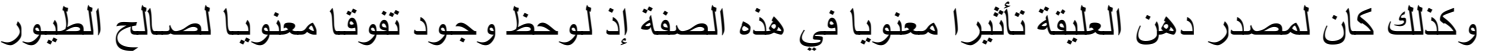

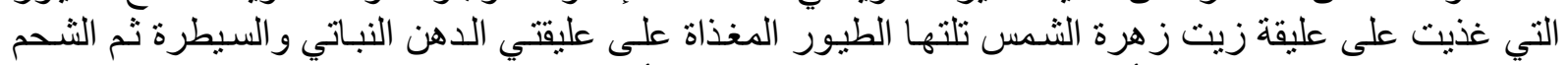

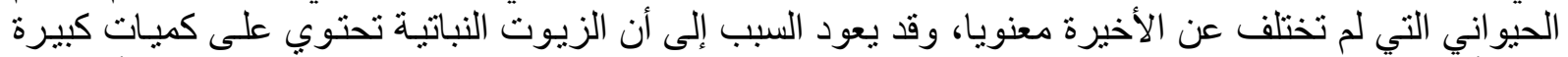
من الأحماض الدهنية غير المشبعة سهلة الهضم والتمثيل مقارنة بالثحوم الحيو النيـة التي تحتوي على التى الأحمـاض

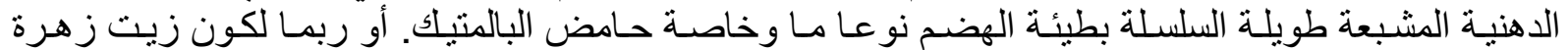

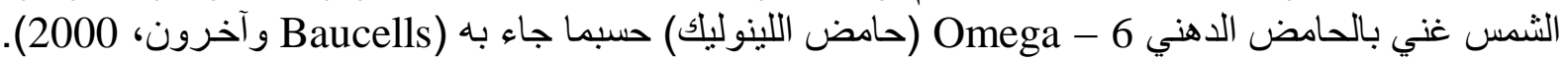
هذا وكان للتناخل بين المجموعة الور اثية ومصدر الدهن تأثثير المعنويا في عدد البيض المنتج إذ إذ أعطت الطيور

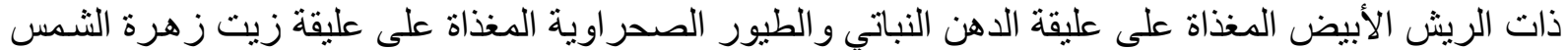

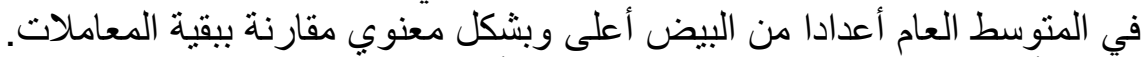

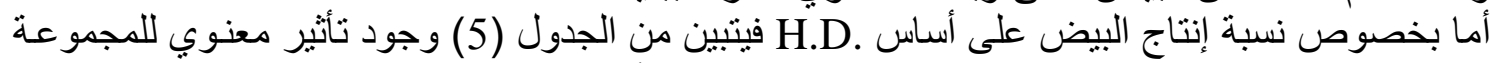

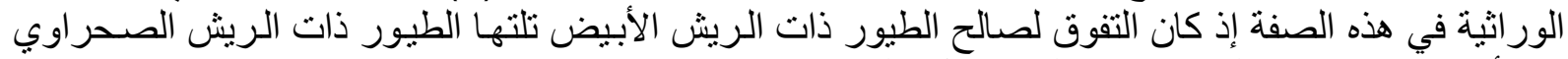
ثم الأسود، جاءت هذه النتائج مؤكدة لما حصل النه عليه (جاسم، 2011). 
Mesopotamia J. of Agric.

Vol. (45) No. (4) 2017
ISSN: 2224 - 9796 (Online)

ISSN: 1815 - $316 \mathrm{X}$ (Print)

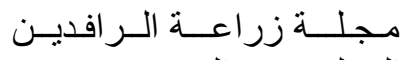

المجلد (45) العدد (4) 2017

الجدول (3): تأثير المجموعة الور اثية ومصدر دهن العليقة والتداخل بينهما في كميات العلف المستهكة (غم/ط).

Table (3): Effect of genetic group, ratio oil source and their interaction on feed consumption $(\mathrm{g} / \mathrm{b})$.

\begin{tabular}{|c|c|c|c|c|c|c|c|}
\hline \multicolumn{6}{|c|}{ الفترات* Periods } & \multirow{2}{*}{\multicolumn{2}{|c|}{ Treatment المعاملة }} \\
\hline Total & 5 & 4 & 3 & 2 & 1 & & \\
\hline \multicolumn{8}{|c|}{ تأتثير المجمو عة الور اثية Genetic group effect } \\
\hline $1994.30 \mathrm{a}$ & 424.72 & $413.60 \mathrm{a}$ & 390.38 & $389.18 \mathrm{a}$ & $376.42 \mathrm{a}$ & \multicolumn{2}{|c|}{ الأبيض White } \\
\hline $1931.96 \mathrm{~b}$ & 419.40 & $401.18 \mathrm{~b}$ & 389.66 & $367.86 \mathrm{c}$ & $353.86 \mathrm{~b}$ & \multicolumn{2}{|c|}{ Black الأسود } \\
\hline $1972.88 \mathrm{a}$ & 428.62 & $409.46 \mathrm{ab}$ & 395.32 & $379.36 \mathrm{~b}$ & $360.12 \mathrm{~b}$ & \multicolumn{2}{|c|}{ الصحر اوي Desert } \\
\hline \multicolumn{8}{|c|}{ Ratio oil source effect تأتيّر مصدر دهن العليقة } \\
\hline $1941.08 \mathrm{~b}$ & 419.44 & 405.84 & 388.48 & $373.52 \mathrm{~b}$ & $353.80 \mathrm{~b}$ & \multicolumn{2}{|c|}{ سيطرة T1 - س م1 } \\
\hline $1990.16 \mathrm{a}$ & 429.16 & 410.12 & 396.46 & $383.26 \mathrm{ab}$ & $371.16 \mathrm{a}$ & \multicolumn{2}{|c|}{ زيت ز هرة الثمس T2 } \\
\hline $1953.32 \mathrm{ab}$ & 426.06 & 408.08 & 387.50 & $373.26 \mathrm{~b}$ & $358.42 \mathrm{ab}$ & \multicolumn{2}{|c|}{ T3ن دانباتي T3 } \\
\hline $1980.96 \mathrm{ab}$ & 422.32 & 408.28 & 394.74 & $385.14 \mathrm{a}$ & $370.46 \mathrm{a}$ & \multicolumn{2}{|c|}{ شيم حيو اني T4 } \\
\hline \multicolumn{8}{|c|}{ Interaction effect (genetic group x ratio oil source)(مصدر دهن العليقة) X تأثير التداخل (المجموعة الور اثنية } \\
\hline $1977.64 \mathrm{ab}$ & 418.58 & $408.96 \mathrm{ab}$ & 384.18 & $391.76 \mathrm{a}$ & $374.16 \mathrm{ab}$ & T1 & \multirow{4}{*}{$\begin{array}{l}\text { الأبيض } \\
\text { White }\end{array}$} \\
\hline $1986.06 \mathrm{a}$ & 423.20 & $417.26 \mathrm{a}$ & 392.00 & $384.92 \mathrm{ab}$ & $368.66 \mathrm{abcd}$ & $\mathrm{T} 2$ & \\
\hline $1991.98 \mathrm{a}$ & 428.08 & $419.24 \mathrm{a}$ & 385.62 & $387.70 \mathrm{ab}$ & $371.34 \mathrm{abc}$ & T3 & \\
\hline $2021.46 \mathrm{a}$ & 429.04 & $408.90 \mathrm{ab}$ & 399.72 & $392.32 \mathrm{a}$ & $391.50 \mathrm{a}$ & T4 & \\
\hline $1902.64 \mathrm{~b}$ & 414.88 & $402.98 \mathrm{ab}$ & 383.78 & $359.34 \mathrm{~cd}$ & $341.66 \mathrm{e}$ & $\mathrm{T} 1$ & \multirow{4}{*}{$\begin{array}{l}\text { الأسود } \\
\text { Black }\end{array}$} \\
\hline $1960.10 \mathrm{ab}$ & 425.16 & $400.24 \mathrm{ab}$ & 395.64 & $375.10 \mathrm{abcd}$ & 363.94 bcde & $\mathrm{T} 2$ & \\
\hline $1898.06 \mathrm{~b}$ & 418.66 & $391.42 \mathrm{~b}$ & 383.90 & $357.52 \mathrm{~d}$ & $346.56 \mathrm{cde}$ & T3 & \\
\hline $1967.04 \mathrm{ab}$ & 418.86 & $410.10 a b$ & 395.36 & $379.46 \mathrm{abc}$ & $363.26 \mathrm{bcde}$ & $\mathrm{T} 4$ & \\
\hline $1942.96 \mathrm{ab}$ & 424.84 & $405.58 \mathrm{ab}$ & 397.46 & $369.48 \mathrm{bcd}$ & $345.58 \mathrm{de}$ & $\mathrm{T} 1$ & \multirow{4}{*}{ الصحراوي } \\
\hline $2024.36 \mathrm{a}$ & 439.12 & $412.88 \mathrm{ab}$ & 401.72 & $389.74 \mathrm{ab}$ & $380.88 \mathrm{ab}$ & $\mathrm{T} 2$ & \\
\hline $1969.90 \mathrm{ab}$ & 431.44 & $413.56 \mathrm{ab}$ & 392.98 & $374.54 \mathrm{abcd}$ & 357.38 bcde & T3 & \\
\hline $1954.34 \mathrm{ab}$ & 419.06 & $405.84 \mathrm{ab}$ & 389.16 & $383.66 \mathrm{ab}$ & 356.64 bcde & $\mathrm{T} 4$ & \\
\hline
\end{tabular}

* مل فترة تمثل 14 يوما.

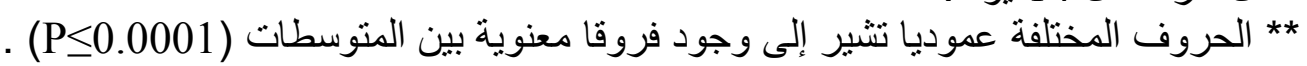

الجدول (4): تأثير المجموعة المظهرية ومصدر دهن العليقة والتداخل بينهما في معدل عدد البيض / طائر. Table (4): Effect of genetic group , ratio oil source and their interaction on average egg number /bird.

\begin{tabular}{|c|c|c|c|c|c|c|c|}
\hline \multicolumn{6}{|c|}{ الفترات * Periods } & \multirow{2}{*}{\multicolumn{2}{|c|}{ Treatment المعاملة }} \\
\hline Total & 5 & 4 & 3 & 2 & 1 & & \\
\hline \multicolumn{8}{|c|}{ Genetic group effect تأثير المجمو عة الور اثية } \\
\hline $50.74 \mathrm{a}$ & $9.90 \mathrm{a}$ & $10.05 \mathrm{a}$ & $10.28 \mathrm{a}$ & $10.24 \mathrm{a}$ & $10.27 \mathrm{a}$ & & الأبيض \\
\hline $38.58 \mathrm{c}$ & $7.02 \mathrm{c}$ & $7.73 \mathrm{c}$ & $7.09 \mathrm{c}$ & $8.63 \mathrm{~b}$ & $8.11 \mathrm{c}$ & & الأسود \\
\hline $46.99 \mathrm{~b}$ & $8.77 \mathrm{~b}$ & $9.12 \mathrm{~b}$ & $9.34 \mathrm{~b}$ & $10.37 \mathrm{a}$ & $9.39 \mathrm{~b}$ & De & الصحر اوي \\
\hline \multicolumn{8}{|c|}{ Ratio oil source effect تأثتير مصدر دهن العليقة } \\
\hline $43.38 \mathrm{bc}$ & $8.62 \mathrm{~b}$ & $8.80 \mathrm{~b}$ & $7.73 \mathrm{c}$ & 9.25 & $8.98 \mathrm{~b}$ & \multicolumn{2}{|c|}{ سيطرة T1 } \\
\hline $50.66 \mathrm{a}$ & $9.40 \mathrm{a}$ & $9.98 \mathrm{a}$ & $10.75 \mathrm{a}$ & 10.18 & $10.35 \mathrm{a}$ & \multicolumn{2}{|c|}{ زيت زهرة الثمس T2 } \\
\hline $44.83 \mathrm{~b}$ & $7.97 \mathrm{~b}$ & $9.24 \mathrm{~b}$ & $8.48 \mathrm{~b}$ & 9.80 & $9.34 \mathrm{~b}$ & \multicolumn{2}{|c|}{ T3 دهن نباتي } \\
\hline $42.18 \mathrm{c}$ & $8.25 \mathrm{~b}$ & $7.84 \mathrm{c}$ & $7.98 \mathrm{c}$ & 9.75 & $8.36 \mathrm{~b}$ & \multicolumn{2}{|c|}{ شحم حيو اني T4 } \\
\hline \multicolumn{8}{|c|}{ Interaction effect (genetic group x ratio oil source)(مصدر دهن العليقة) X تأثير التداخل (المجموعة الور اثئة } \\
\hline $47.08 \mathrm{bc}$ & $9.45 \mathrm{bc}$ & $9.77 \mathrm{c}$ & $8.67 \mathrm{~cd}$ & $9.39 \mathrm{bcd}$ & $9.80 \mathrm{a}$ & $\mathrm{T} 1$ & \multirow{4}{*}{ الأبيض } \\
\hline $50.68 \mathrm{~b}$ & $9.22 \mathrm{bc}$ & $10.33 \mathrm{bc}$ & $11.20 \mathrm{a}$ & $9.18 \mathrm{~cd}$ & $10.73 \mathrm{a}$ & $\mathrm{T} 2$ & \\
\hline $55.16 \mathrm{a}$ & $11.11 \mathrm{a}$ & $11.42 \mathrm{a}$ & $10.07 \mathrm{a}$ & $11.58 \mathrm{a}$ & $10.98 \mathrm{a}$ & T3 & \\
\hline $48.06 \mathrm{bc}$ & $9.82 \mathrm{~b}$ & $8.68 \mathrm{de}$ & $9.17 \mathrm{bc}$ & $10.80 \mathrm{abc}$ & $9.58 \mathrm{abc}$ & T4 & \\
\hline $41.20 \mathrm{~d}$ & $7.33 \mathrm{de}$ & $8.53 \mathrm{de}$ & $6.46 \mathrm{fg}$ & $9.28 \mathrm{~cd}$ & $9.60 \mathrm{abc}$ & $\mathrm{T} 1$ & \multirow{3}{*}{ الأسود } \\
\hline $45.02 \mathrm{~cd}$ & $9.13 \mathrm{bc}$ & $8.61 \mathrm{de}$ & $9.06 \mathrm{bc}$ & $9.33 \mathrm{bcd}$ & $8.89 \mathrm{bcd}$ & $\mathrm{T} 2$ & \\
\hline $31.01 \mathrm{f}$ & $5.14 \mathrm{f}$ & $6.87 \mathrm{f}$ & $5.58 \mathrm{~g}$ & $6.83 \mathrm{e}$ & $6.59 \mathrm{e}$ & T3 & \\
\hline
\end{tabular}


Mesopotamia J. of Agric.

Vol. (45) No. (4) 2017
ISSN: 2224 - 9796 (Online)

ISSN: 1815 - $316 \mathrm{X}$ (Print)

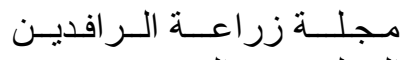

المجلد (45) العدد (4) 2017

\begin{tabular}{|c|c|c|c|c|c|c|c|}
\hline \multicolumn{6}{|c|}{ الفترات* Periods } & \multirow{2}{*}{\multicolumn{2}{|c|}{ Treatment المعاملة }} \\
\hline Total & 5 & 4 & 3 & 2 & 1 & & \\
\hline $37.07 \mathrm{e}$ & $6.47 \mathrm{e}$ & $6.91 \mathrm{f}$ & $7.25 \mathrm{ef}$ & $9.07 \mathrm{~d}$ & $7.37 \mathrm{de}$ & T4 & \\
\hline $41.86 \mathrm{~d}$ & $9.07 \mathrm{bc}$ & $8.09 \mathrm{e}$ & $8.07 \mathrm{de}$ & $9.08 \mathrm{~d}$ & $7.55 \mathrm{de}$ & $\mathrm{T} 1$ & الصحر اوي \\
\hline $56.30 \mathrm{a}$ & $9.85 \mathrm{~b}$ & $11.00 \mathrm{ab}$ & $12.00 \mathrm{a}$ & $12.03 \mathrm{a}$ & $11.42 \mathrm{a}$ & $\mathrm{T} 2$ & Desert \\
\hline $48.31 \mathrm{bc}$ & $7.67 \mathrm{~d}$ & $9.44 \mathrm{~cd}$ & $9.78 \mathrm{~b}$ & $10.98 \mathrm{ab}$ & $10.44 \mathrm{ab}$ & T3 & \\
\hline $41.42 \mathrm{~d}$ & $8.47 \mathrm{~cd}$ & $7.93 \mathrm{e}$ & $7.51 \mathrm{e}$ & $9.38 \mathrm{bcd}$ & $8.13 \mathrm{cde}$ & T4 & \\
\hline
\end{tabular}

* * الحروف المختلفة عموديا تثير إلى وجود فروقا معنوية بين المنوسطات (p<0.0001).

الجدول (5): تأثثر المجمو عة الور اثية ومصدر دهن العليقة والتداخل بينهما في إنتاج البيض \% (H.D). Table (5): Effect of genetic group, ratio oil source and their interaction on egg production \% (H.D)

\begin{tabular}{|c|c|c|c|c|c|c|c|}
\hline \multicolumn{6}{|c|}{ Periods * الفترات } & \multirow{2}{*}{\multicolumn{2}{|c|}{ Treatment المعاملة }} \\
\hline $\begin{array}{c}\text { General } \\
\text { mean }\end{array}$ & 5 & 4 & 3 & 2 & 1 & & \\
\hline \multicolumn{8}{|c|}{ تأثنير المجموعة الور اثية Genetic group effect } \\
\hline $72.48 \mathrm{a}$ & $70.70 \mathrm{a}$ & $71.82 \mathrm{a}$ & $73.39 \mathrm{a}$ & $73.12 \mathrm{a}$ & $73.38 \mathrm{a}$ & \multicolumn{2}{|c|}{ الأبيض White } \\
\hline $55.11 \mathrm{c}$ & $50.15 \mathrm{c}$ & $55.21 \mathrm{c}$ & $50.62 \mathrm{c}$ & $61.64 \mathrm{~b}$ & $67.05 \mathrm{~b}$ & \multicolumn{2}{|c|}{ الأسود Black } \\
\hline $67.11 \mathrm{~b}$ & $62.59 \mathrm{~b}$ & $65.12 \mathrm{~b}$ & $66.72 \mathrm{~b}$ & $74.05 \mathrm{a}$ & $57.94 \mathrm{c}$ & \multicolumn{2}{|c|}{ الصحر اوي Desert } \\
\hline \multicolumn{8}{|c|}{ Ratio oil source effect تأثير مصدر دهن العليقة } \\
\hline $61.97 \mathrm{bc}$ & $61.54 \mathrm{~b}$ & $62.83 \mathrm{~b}$ & $55.22 \mathrm{c}$ & 66.06 & $64.18 \mathrm{~b}$ & \multicolumn{2}{|c|}{ T1 سيطرة T1 } \\
\hline $72.38 \mathrm{a}$ & $67.16 \mathrm{a}$ & $71.30 \mathrm{a}$ & $76.80 \mathrm{a}$ & 72.73 & $73.90 \mathrm{a}$ & \multicolumn{2}{|c|}{ زيت زهرة الشمس T2 } \\
\hline $64.99 \mathrm{~b}$ & $56.95 \mathrm{~b}$ & $66.05 \mathrm{~b}$ & $65.30 \mathrm{~b}$ & 69.97 & $66.69 \mathrm{~b}$ & \multicolumn{2}{|c|}{ Tد دهن نباتي T3 } \\
\hline $60.26 \mathrm{c}$ & $58.94 \mathrm{~b}$ & $56.02 \mathrm{c}$ & $56.98 \mathrm{c}$ & 69.64 & $59.73 \mathrm{~b}$ & \multicolumn{2}{|c|}{ شحم حيواني T4 } \\
\hline \multicolumn{8}{|c|}{ Interaction effect (genetic group x ratio oil source)(مصدر دهن العليقة) X تأثير التداخل (الهموعة الور اثثة } \\
\hline $67.26 \mathrm{bc}$ & $67.46 \mathrm{bc}$ & $69.84 \mathrm{c}$ & $61.90 \mathrm{~cd}$ & $67.06 \mathrm{bcd}$ & $4.90 \mathrm{abc}$ & $\mathrm{T} 1$ & \multirow{4}{*}{$\begin{array}{l}\text { الأبيض } \\
\text { White }\end{array}$} \\
\hline $72.38 \mathrm{~b}$ & $65.87 \mathrm{bc}$ & $73.81 \mathrm{bc}$ & $80.00 \mathrm{a}$ & $65.56 \mathrm{~cd}$ & $5.37 \mathrm{a}$ & $\mathrm{T} 2$ & \\
\hline $81.65 \mathrm{a}$ & $79.36 \mathrm{a}$ & $81.58 \mathrm{a}$ & $86.19 \mathrm{a}$ & $82.70 \mathrm{a}$ & $5.49 \mathrm{a}$ & T3 & \\
\hline $68.64 \mathrm{bc}$ & $70.12 \mathrm{~b}$ & $62.02 \mathrm{de}$ & $65.48 \mathrm{bc}$ & $77.14 \mathrm{abc}$ & $4.79 \mathrm{abc}$ & $\mathrm{T} 4$ & \\
\hline $58.84 \mathrm{~d}$ & $52.38 \mathrm{de}$ & $60.87 \mathrm{de}$ & $46.11 \mathrm{fg}$ & $66.27 \mathrm{~cd}$ & $4.80 \mathrm{abc}$ & $\mathrm{T} 1$ & \multirow{4}{*}{$\begin{array}{l}\text { الأسود } \\
\text { Black }\end{array}$} \\
\hline $64.32 \mathrm{~cd}$ & $65.24 \mathrm{bc}$ & $61.51 \mathrm{de}$ & $64.69 \mathrm{bc}$ & $66.67 \mathrm{~cd}$ & $4.44 \mathrm{bcd}$ & $\mathrm{T} 2$ & \\
\hline $44.31 \mathrm{f}$ & $36.73 \mathrm{f}$ & $49.09 \mathrm{f}$ & $39.86 \mathrm{~g}$ & $48.81 \mathrm{e}$ & $3.29 \mathrm{e}$ & T3 & \\
\hline $52.97 \mathrm{e}$ & $46.23 \mathrm{e}$ & $49.36 \mathrm{f}$ & 51.82 ef & $64.80 \mathrm{~d}$ & $3.69 \mathrm{de}$ & $\mathrm{T} 4$ & \\
\hline $59.80 \mathrm{~d}$ & $64.76 \mathrm{~cd}$ & $57.78 \mathrm{e}$ & $57.66 \mathrm{de}$ & $64.84 \mathrm{~d}$ & $3.78 \mathrm{de}$ & T1 & \multirow{4}{*}{ الصحراوي } \\
\hline $80.43 \mathrm{a}$ & $70.36 \mathrm{~b}$ & $78.57 \mathrm{ab}$ & $85.71 \mathrm{a}$ & $85.95 \mathrm{a}$ & $5.71 \mathrm{a}$ & $\mathrm{T} 2$ & \\
\hline $69.02 \mathrm{bc}$ & $54.76 \mathrm{~d}$ & $67.46 \mathrm{~cd}$ & $69.84 \mathrm{~b}$ & $78.42 \mathrm{ab}$ & $5.22 \mathrm{ab}$ & $\mathrm{T} 3$ & \\
\hline $59.18 \mathrm{~d}$ & $60.48 \mathrm{~cd}$ & $56.66 \mathrm{e}$ & $53.65 \mathrm{e}$ & $66.99 \mathrm{bcd}$ & $4.07 \mathrm{cde}$ & $\mathrm{T} 4$ & \\
\hline
\end{tabular}

* مل فترة تمنل 14 يوما.

* * الحروف المختلفة عموديا تشير إلى وجود فروقا معنوية بين المتوسطات (p

وكذلك ظهر لمصدر الدهن تأثير اً معنوياً في نسبة إنتاج البيض إذ تفوقت الطيور المغذاة على عليقة زيت

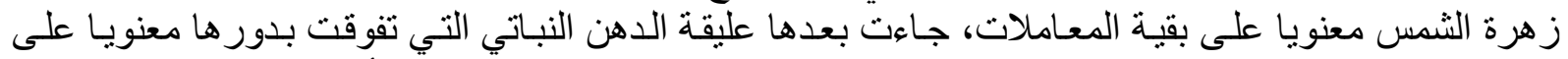

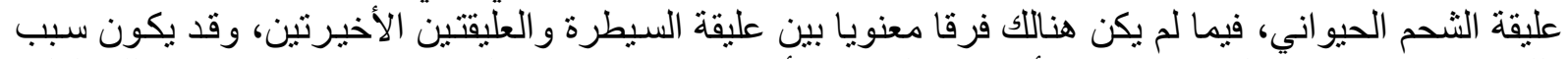

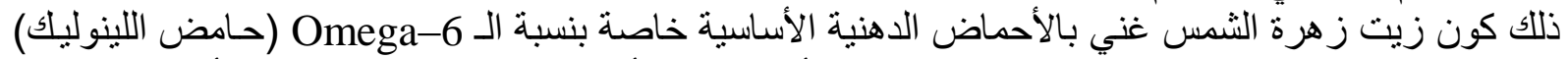

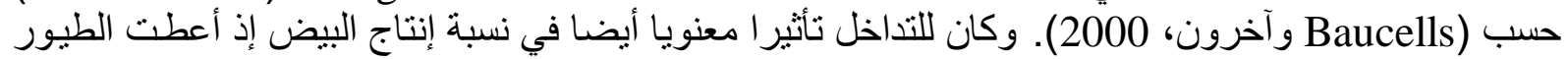

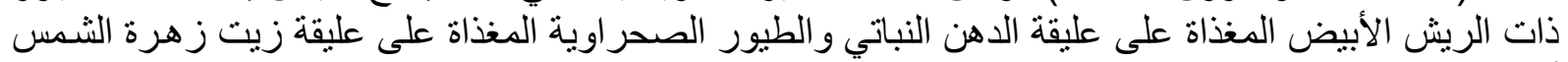

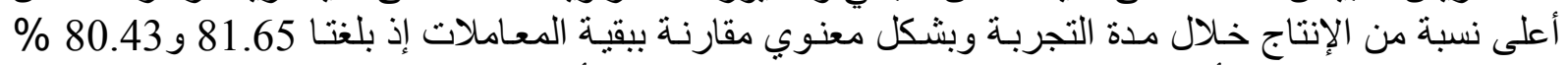

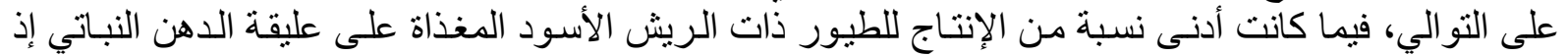

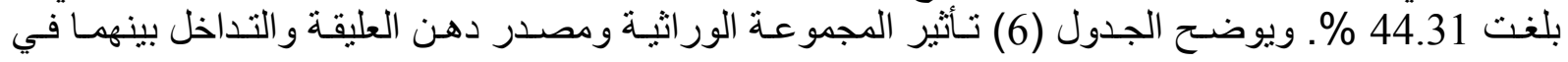


Mesopotamia J. of Agric.

Vol. (45) No. (4) 2017
ISSN: 2224 - 9796 (Online)

ISSN: 1815 - $316 \mathrm{X}$ (Print)

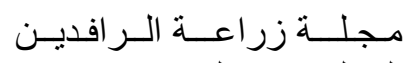

المجلد (45) العدد (4) 2017

متوسطوزن البيضة (غم) إذ تبيين عدم وجود تاثثير معنوي للمجموعة الور اثيـة ومصدر دهن العليقة والتداخل

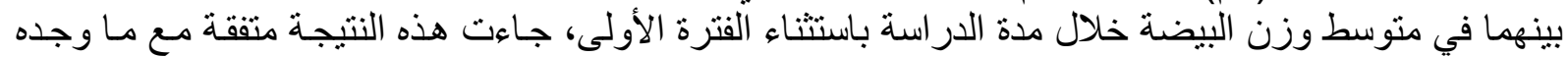

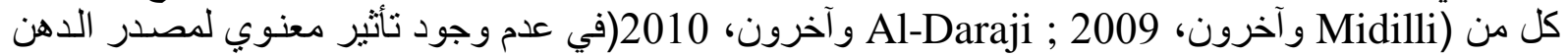
بالعليقة في متوسط وزن البيضة.

الجدول (6): تأثير المجموعة الور اثية ومصدر دهن العليقة والتداخل بينهما في متوسطوزن البيضة (غم) Table (6): Effect of genetic group, ratio oil source and their interaction on egg weight mean $(\mathrm{g})$.

\begin{tabular}{|c|c|c|c|c|c|c|c|}
\hline \multicolumn{6}{|c|}{ الفترات* Periods } & \multirow{2}{*}{\multicolumn{2}{|c|}{ Treatment المعاملة }} \\
\hline General mean & 5 & 4 & 3 & 2 & 1 & & \\
\hline \multicolumn{8}{|c|}{ تأتثير المجموعة الور اثية Genetic group effect } \\
\hline 11.95 & 12.08 & 12.24 & 12.14 & 11.85 & $11.43 \mathrm{ab}$ & \multicolumn{2}{|c|}{ الأبيض White } \\
\hline 11.59 & 11.97 & 11.82 & 11.73 & 11.47 & $10.97 \mathrm{~b}$ & \multicolumn{2}{|c|}{ الأسود Black } \\
\hline 12.05 & 12.35 & 12.24 & 12.26 & 11.92 & $11.59 \mathrm{a}$ & \multicolumn{2}{|c|}{ الصحر اوي Desert } \\
\hline \multicolumn{8}{|c|}{ تأَثير مصدر دهن العليقة Ratio oil source effect } \\
\hline 11.68 & 12.05 & 12.12 & 11.68 & 11.37 & 11.20 & \multicolumn{2}{|c|}{ سيطرة T1 } \\
\hline 12.01 & 12.19 & 11.89 & 12.17 & 12.03 & 11.76 & \multicolumn{2}{|c|}{ زيت زهرة الثمس T2 } \\
\hline 11.97 & 12.25 & 12.09 & 12.36 & 11.94 & 11.20 & \multicolumn{2}{|c|}{ T3 دهن نباتى T3 } \\
\hline 11.79 & 12.03 & 12.13 & 11.96 & 11.65 & 11.16 & \multicolumn{2}{|c|}{ شحم حيو اني T4 } \\
\hline \multicolumn{8}{|c|}{ Interaction effect (genetic group x ratio oil source)(مصدر دهن العليقة) X نأثير التداخل (المجموعة الور اثثية } \\
\hline 11.55 & 11.82 & 12.21 & 11.30 & 11.18 & $11.23 \mathrm{ab}$ & T1 & \multirow{4}{*}{$\begin{array}{l}\text { الأبيض } \\
\text { White }\end{array}$} \\
\hline 12.30 & 12.04 & 12.35 & 12.72 & 12.42 & $12.00 \mathrm{a}$ & $\mathrm{T} 2$ & \\
\hline 12.24 & 12.65 & 12.48 & 12.58 & 12.21 & $11.30 \mathrm{ab}$ & T3 & \\
\hline 11.69 & 11.80 & 11.91 & 11.96 & 11.60 & $11.20 \mathrm{ab}$ & T4 & \\
\hline 11.47 & 11.84 & 11.67 & 11.56 & 11.24 & $11.06 \mathrm{ab}$ & $\mathrm{T} 1$ & \multirow{4}{*}{$\begin{array}{l}\text { الأسود } \\
\text { Black }\end{array}$} \\
\hline 11.67 & 11.99 & 11.58 & 11.69 & 11.76 & $11.34 \mathrm{ab}$ & $\mathrm{T} 2$ & \\
\hline 11.49 & 12.04 & 11.90 & 11.89 & 11.24 & $10.37 \mathrm{~b}$ & T3 & \\
\hline 11.74 & 12.00 & 12.14 & 11.79 & 11.65 & $11.12 \mathrm{ab}$ & T4 & \\
\hline 12.03 & 12.51 & 12.46 & 12.19 & 11.69 & $11.32 \mathrm{ab}$ & $\mathrm{T} 1$ & \multirow{4}{*}{ الصحر اوي } \\
\hline 12.05 & 12.53 & 11.74 & 12.11 & 11.91 & $11.95 \mathrm{a}$ & $\mathrm{T} 2$ & \\
\hline 12.17 & 12.04 & 11.89 & 12.61 & 12.38 & $11.94 \mathrm{a}$ & T3 & \\
\hline 11.93 & 12.30 & 12.36 & 12.13 & 11.70 & $11.15 \mathrm{ab}$ & $\mathrm{T} 4$ & \\
\hline
\end{tabular}
* * الحروف المختلفة عموديا تثير إلى وجود فروقا معنوية بين المتوسطات (p<0.0001).

أما بخصوص كفاءة التحويل الغذائي فيثير الجدول (7) إلى وجود تأثنير معنوي للمجمو عة الور اثيـة في

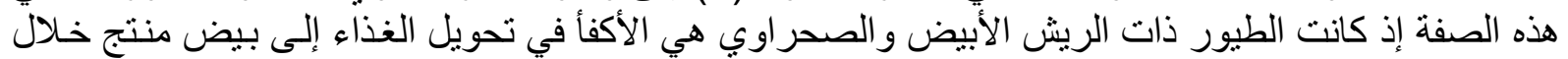

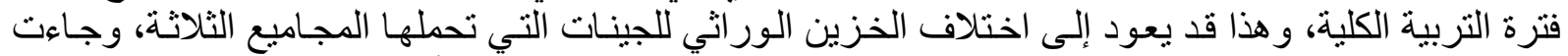

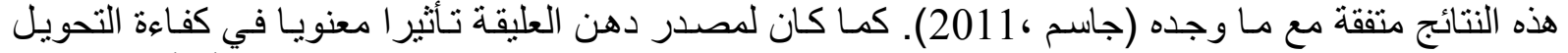

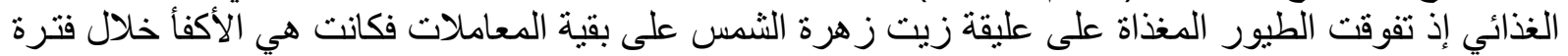

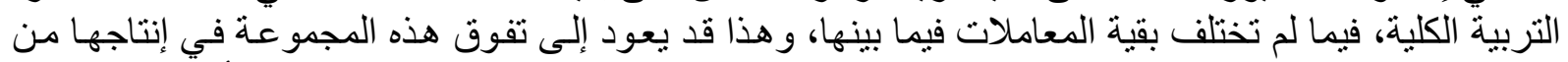

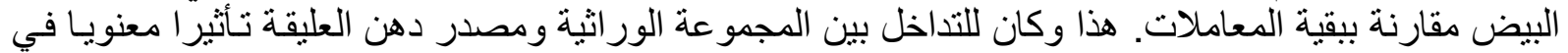

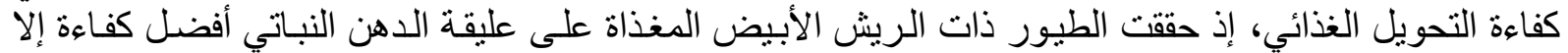

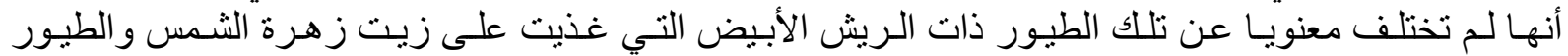

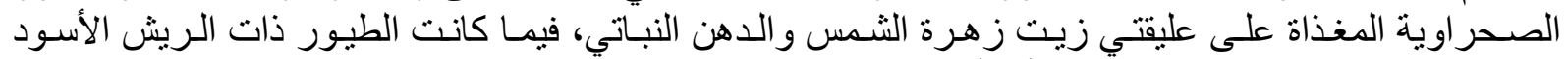

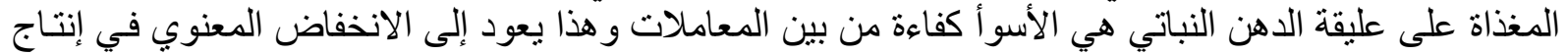

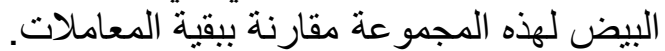


الجدول (7): تأثثير المجموعة الور اثيـة ومصدر دهن العليقة والتداخل بينهمـا في معامل التحويل الغذائي (غم

Table (7): Effect of genetic group , ratio oil source and their interaction on feed conversion (g.feed/g.egg)

\begin{tabular}{|c|c|c|c|c|c|c|c|}
\hline \multicolumn{6}{|c|}{ الفترات * Periods } & \multirow{2}{*}{\multicolumn{2}{|c|}{ Treatment المعاملة }} \\
\hline $\begin{array}{c}\text { General } \\
\text { mean }\end{array}$ & 5 & 4 & 3 & 2 & 1 & & \\
\hline & \multicolumn{7}{|c|}{ تأتثير المجموعة الور اثية Genetic group effect } \\
\hline $3.35 \mathrm{~b}$ & $3.59 \mathrm{c}$ & $3.41 \mathrm{c}$ & $3.22 \mathrm{c}$ & $3.27 \mathrm{~b}$ & $3.27 \mathrm{~b}$ & \multicolumn{2}{|c|}{ الأبيض White } \\
\hline $4.49 \mathrm{a}$ & $5.23 \mathrm{a}$ & $4.46 \mathrm{a}$ & $4.85 \mathrm{a}$ & $3.82 \mathrm{a}$ & $4.13 \mathrm{a}$ & \multicolumn{2}{|c|}{ الأسود Black } \\
\hline $3.55 \mathrm{~b}$ & $4.01 \mathrm{~b}$ & $3.58 \mathrm{~b}$ & $3.59 \mathrm{~b}$ & $3.15 \mathrm{~b}$ & $3.44 \mathrm{~b}$ & \multicolumn{2}{|c|}{ الصحر اوي Desert } \\
\hline & \multicolumn{7}{|c|}{ Ratio oil source effect أثنير مصدر دهن العليقة } \\
\hline $3.90 \mathrm{a}$ & $4.10 \mathrm{~b}$ & $3.84 \mathrm{~b}$ & $4.39 \mathrm{a}$ & 3.58 & $3.59 \mathrm{ab}$ & \multicolumn{2}{|c|}{ 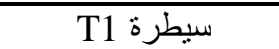 } \\
\hline $3.34 \mathrm{~b}$ & $3.77 \mathrm{c}$ & $3.52 \mathrm{~b}$ & $3.09 \mathrm{c}$ & 3.19 & $3.11 \mathrm{~b}$ & \multicolumn{2}{|c|}{ زيت زهرة الثمسT2 } \\
\hline $3.92 \mathrm{a}$ & $4.85 \mathrm{a}$ & $3.82 \mathrm{~b}$ & $3.85 \mathrm{~b}$ & 3.41 & $3.67 \mathrm{a}$ & \multicolumn{2}{|c|}{ د3ن دهن نباتي T3 } \\
\hline $4.05 \mathrm{a}$ & $4.39 \mathrm{~b}$ & $4.10 \mathrm{a}$ & $4.22 \mathrm{a}$ & 3.46 & $4.07 \mathrm{a}$ & \multicolumn{2}{|c|}{ شحم حيواني T4 } \\
\hline \multicolumn{8}{|c|}{ Interaction effect (genetic group x ratio oil source)(مصدر دهن العليقة) X تأثير التخاخل (المجموعة الور اثثية } \\
\hline $3.66 \mathrm{cde}$ & $3.75 \mathrm{~d}$ & 3.43 cde & $3.93 \mathrm{~d}$ & $3.75 \mathrm{~b}$ & 3.45 cde & $\mathrm{T} 1$ & \multirow{4}{*}{$\begin{array}{l}\text { الأبيض } \\
\text { White }\end{array}$} \\
\hline 3.23 ef & $3.82 \mathrm{~d}$ & $3.28 \mathrm{de}$ & $2.76 \mathrm{f}$ & $3.41 \mathrm{bc}$ & $2.89 \mathrm{e}$ & $\mathrm{T} 2$ & \\
\hline $2.88 \mathrm{f}$ & $3.08 \mathrm{e}$ & $2.96 \mathrm{e}$ & $2.54 \mathrm{f}$ & $2.76 \mathrm{c}$ & $3.04 \mathrm{de}$ & T3 & \\
\hline 3.64 cde & $3.71 \mathrm{~d}$ & $3.97 \mathrm{bc}$ & 3.66de & $3.16 \mathrm{bc}$ & 3.69 bcde & T4 & \\
\hline $4.15 \mathrm{bc}$ & $4.80 \mathrm{c}$ & $4.05 \mathrm{bc}$ & $5.17 \mathrm{ab}$ & $3.47 \mathrm{bc}$ & $3.25 \mathrm{cde}$ & T1 & \multirow{4}{*}{$\begin{array}{l}\text { الأسود } \\
\text { Black }\end{array}$} \\
\hline 3.77 cde & $3.92 \mathrm{~d}$ & $4.07 \mathrm{bc}$ & $3.75 \mathrm{de}$ & $3.44 \mathrm{bc}$ & 3.65 bcde & $\mathrm{T} 2$ & \\
\hline $5.44 \mathrm{a}$ & $6.79 \mathrm{a}$ & $4.81 \mathrm{a}$ & $5.82 \mathrm{a}$ & $4.69 \mathrm{a}$ & $5.09 \mathrm{a}$ & T3 & \\
\hline $4.65 \mathrm{~b}$ & $5.41 \mathrm{~b}$ & $4.94 \mathrm{a}$ & $4.69 \mathrm{bc}$ & $3.68 \mathrm{~b}$ & $4.51 \mathrm{~b}$ & $\mathrm{~T} 4$ & \\
\hline $3.90 \mathrm{~cd}$ & $3.75 \mathrm{~d}$ & $4.05 \mathrm{cb}$ & $4.08 \mathrm{~cd}$ & $3.53 \mathrm{bc}$ & $4.07 \mathrm{bc}$ & $\mathrm{T} 1$ & \multirow{4}{*}{ الصحراوي } \\
\hline $3.01 \mathrm{f}$ & $3.56 \mathrm{de}$ & $3.20 \mathrm{de}$ & $2.77 \mathrm{f}$ & $2.73 \mathrm{c}$ & $2.80 \mathrm{e}$ & $\mathrm{T} 2$ & \\
\hline $3.44 \mathrm{def}$ & $4.68 \mathrm{c}$ & $3.68 \mathrm{bcd}$ & $3.20 \mathrm{ef}$ & $2.78 \mathrm{c}$ & $2.87 \mathrm{e}$ & T3 & \\
\hline $3.86 \mathrm{~cd}$ & $4.05 \mathrm{~d}$ & $3.39 \mathrm{~b}$ & $4.31 \mathrm{~cd}$ & $3.55 \mathrm{bc}$ & $4.00 \mathrm{bcd}$ & $\mathrm{T} 4$ & \\
\hline
\end{tabular}

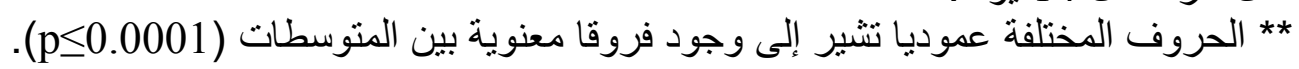

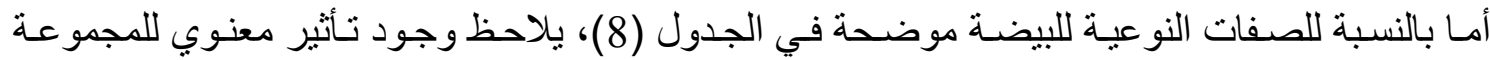

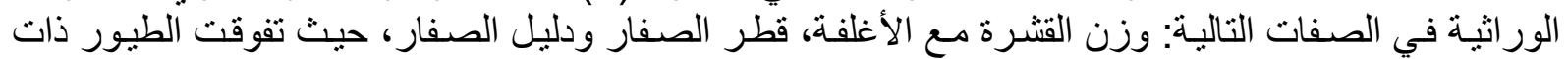
الريش الأبيض والصحر اوي معنويا على الطيور ذات الريش الأسود في صفتي وزن القترة وقطر الصفر الصفار، في في

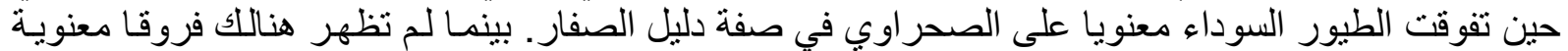

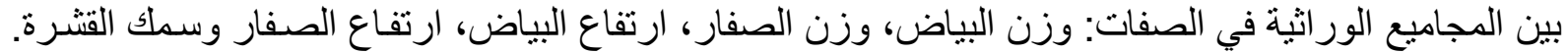

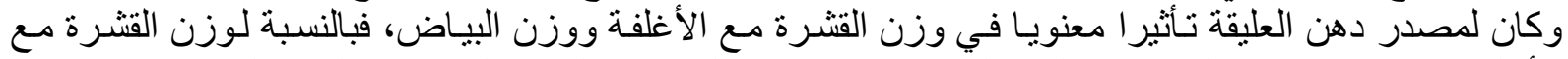

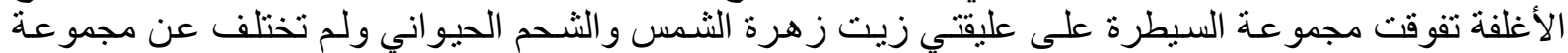

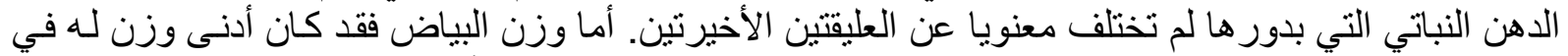

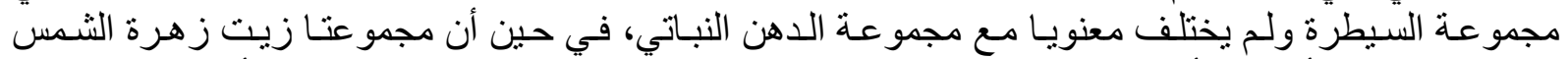

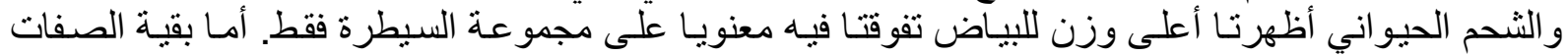
النوعية فلم يظهر لمصدر دهن العليقة تأثثير المعنويا فيها. 
Mesopotamia J. of Agric.

Vol. (45) No. (4) 2017
ISSN: 2224 - 9796 (Online)

ISSN: 1815 - 316 X (Print)

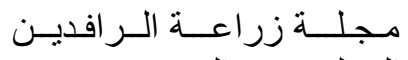

المجلد (45) العدد (4) 2017

الجدول (8): تأثثر المجموعة الور اثية ومصدر دهن العليقة والتداخل بينهما في بعض الصفات النوعية للبيض.

Table (8): Effect of genetic group , ratio oil source and their interaction on egg quality traits.

\begin{tabular}{|c|c|c|c|c|c|c|c|c|c|}
\hline \multicolumn{8}{|c|}{ الصفات Traits } & \multirow{2}{*}{\multicolumn{2}{|c|}{ Treatment المعاملات }} \\
\hline $\operatorname{Tr}_{8}$ & $\operatorname{Tr}_{7}$ & $\operatorname{Tr}_{6}$ & $\operatorname{Tr}_{5}$ & $\operatorname{Tr}_{4}$ & $\operatorname{Tr}_{3}$ & $\operatorname{Tr}_{2}$ & $\operatorname{Tr}_{1}$ & & \\
\hline \multicolumn{10}{|c|}{ تأثيّر المجموعة الور اثية } \\
\hline 0.38 & $0.48 \mathrm{ab}$ & $25.46 \mathrm{a}$ & 12.33 & 3.82 & 4.03 & 5.82 & $1.78 \mathrm{a}$ & \multicolumn{2}{|c|}{ الأبيض White } \\
\hline 0.37 & $0.49 \mathrm{a}$ & $24.67 \mathrm{~b}$ & 12.14 & 3.77 & 3.86 & 5.68 & $1.65 \mathrm{~b}$ & \multicolumn{2}{|c|}{ الأسود Black } \\
\hline 0.36 & $0.47 \mathrm{~b}$ & $25.49 \mathrm{a}$ & 12.10 & 3.90 & 3.92 & 5.88 & $1.75 \mathrm{ab}$ & \multicolumn{2}{|c|}{ الصحر اوي Desert } \\
\hline \multicolumn{10}{|c|}{ Ratio oil source effect تأثيثير مصدر دهن العليقة } \\
\hline 0.35 & 0.49 & 25.03 & 12.35 & 3.81 & 3.97 & $5.39 \mathrm{~b}$ & $1.82 \mathrm{a}$ & \multicolumn{2}{|c|}{ سيطرة T1 } \\
\hline 0.38 & 0.49 & 25.04 & 12.20 & 3.84 & 3.88 & $6.07 \mathrm{a}$ & $1.67 \mathrm{~b}$ & \multicolumn{2}{|c|}{ زيت زهرة الثمس T2 } \\
\hline 0.36 & 0.48 & 25.55 & 12.16 & 3.80 & 4.07 & $5.70 \mathrm{ab}$ & $1.76 \mathrm{ab}$ & \multicolumn{2}{|c|}{ د3 دهن نباتي T3 } \\
\hline 0.38 & 0.48 & 25.21 & 12.04 & 3.88 & 3.82 & $5.93 \mathrm{a}$ & $1.68 \mathrm{~b}$ & \multicolumn{2}{|c|}{ شحم حيو اني T4 } \\
\hline \multicolumn{10}{|c|}{ interaction effect (genetic group x ratio oil source)(مصدر دهن العليقة) x تأثتير التداخل (المجموعة الور اثثية) } \\
\hline $0.39 \mathrm{ab}$ & $0.50 \mathrm{a}$ & $25.03 \mathrm{abc}$ & 12.59 & $3.96 \mathrm{a}$ & 3.90 & $5.30 \mathrm{~b}$ & $1.88 \mathrm{ab}$ & $\mathrm{T} 1$ & \multirow{4}{*}{$\begin{array}{l}\text { الأبيض } \\
\text { White }\end{array}$} \\
\hline $0.39 \mathrm{ab}$ & $0.50 \mathrm{a}$ & $25.26 a b c$ & 12.52 & $3.73 \mathrm{ab}$ & 4.13 & $6.19 \mathrm{a}$ & $1.72 \mathrm{abc}$ & $\mathrm{T} 2$ & \\
\hline $0.40 \mathrm{ab}$ & $0.48 \mathrm{ab}$ & $25.92 \mathrm{ab}$ & 12.44 & $3.73 \mathrm{ab}$ & 4.20 & $5.72 \mathrm{ab}$ & $1.90 \mathrm{a}$ & T3 & \\
\hline $0.34 \mathrm{ab}$ & $0.46 \mathrm{~b}$ & $25.59 \mathrm{abc}$ & 11.76 & $3.90 \mathrm{ab}$ & 3.84 & $5.91 \mathrm{ab}$ & $1.65 \mathrm{abc}$ & $\mathrm{T} 4$ & \\
\hline $0.32 \mathrm{~b}$ & $0.49 \mathrm{ab}$ & $24.53 \mathrm{bc}$ & 12.00 & $3.54 \mathrm{~b}$ & 4.00 & $5.25 \mathrm{~b}$ & $1.76 \mathrm{abc}$ & $\mathrm{T} 1$ & \multirow{4}{*}{$\begin{array}{l}\text { الأسود } \\
\text { Black }\end{array}$} \\
\hline $0.38 \mathrm{ab}$ & $0.49 \mathrm{ab}$ & $24.93 \mathrm{abc}$ & 12.23 & $3.93 \mathrm{ab}$ & 3.79 & $6.17 \mathrm{a}$ & $1.61 \mathrm{bc}$ & $\mathrm{T} 2$ & \\
\hline $0.36 \mathrm{ab}$ & $0.50 \mathrm{a}$ & $24.42 \mathrm{c}$ & 12.13 & $3.75 \mathrm{ab}$ & 3.81 & $5.56 \mathrm{ab}$ & $1.59 \mathrm{c}$ & T3 & \\
\hline $0.42 \mathrm{a}$ & $0.49 \mathrm{ab}$ & $24.73 \mathrm{abc}$ & 12.19 & $3.82 \mathrm{ab}$ & 3.86 & $5.47 \mathrm{ab}$ & $1.69 \mathrm{abc}$ & $\mathrm{T} 4$ & \\
\hline $0.34 \mathrm{ab}$ & $0.49 \mathrm{ab}$ & $25.66 \mathrm{abc}$ & 12.53 & $4.01 \mathrm{a}$ & 4.00 & $5.64 \mathrm{ab}$ & $1.85 \mathrm{abc}$ & $\mathrm{T} 1$ & \multirow{4}{*}{ الصحراوي } \\
\hline $0.38 \mathrm{ab}$ & $0.47 \mathrm{ab}$ & $24.95 \mathrm{abc}$ & 11.83 & $3.82 \mathrm{ab}$ & 3.73 & $5.80 \mathrm{ab}$ & $1.68 \mathrm{abc}$ & $\mathrm{T} 2$ & \\
\hline $0.33 \mathrm{~b}$ & $0.46 \mathrm{~b}$ & $26.14 \mathrm{a}$ & 11.97 & $3.90 \mathrm{ab}$ & 4.17 & $5.80 \mathrm{ab}$ & $1.78 \mathrm{abc}$ & T3 & \\
\hline $0.40 \mathrm{ab}$ & $0.49 \mathrm{ab}$ & $25.14 a b c$ & 12.20 & $3.90 \mathrm{ab}$ & 3.78 & $6.21 \mathrm{a}$ & $1.69 \mathrm{abc}$ & $\mathrm{T} 4$ & \\
\hline
\end{tabular}

الحروف المختلفة عموديا تشير إلى وجود فروقا معنوية بين المتوسطات (6.21 (p0.0001).

إذ أن ،(albumin weight)

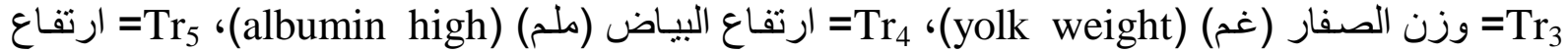
،(yolk high) (م فار (م) (م) (م)

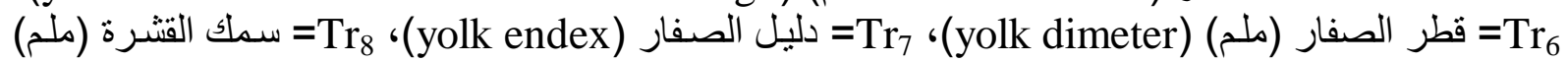
.(shell thickness)

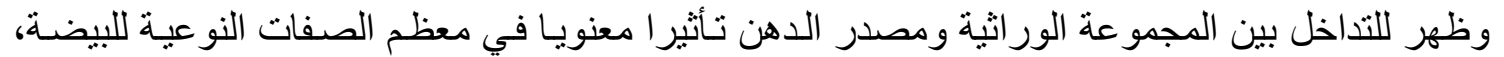

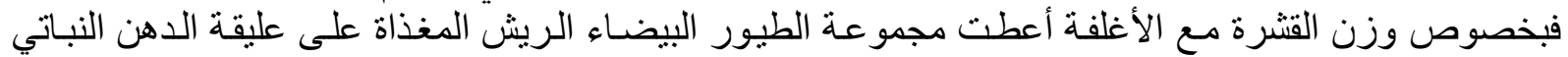

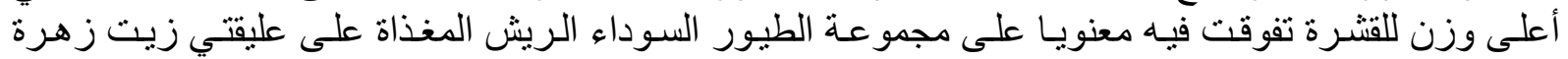

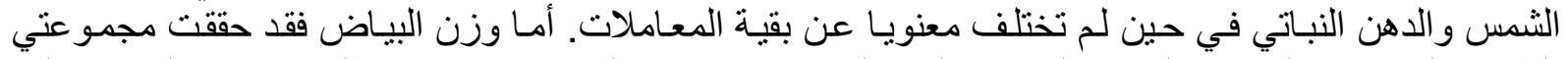

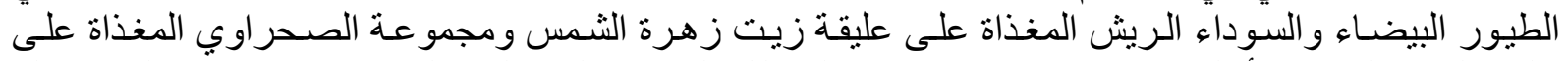

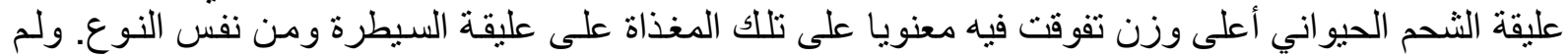

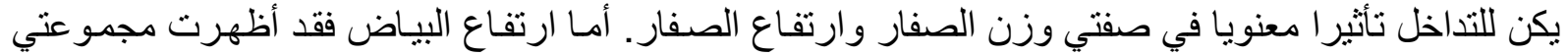

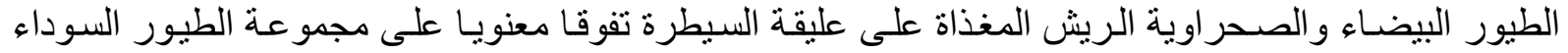

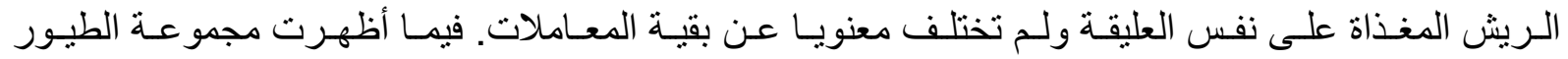

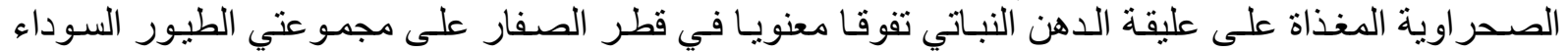

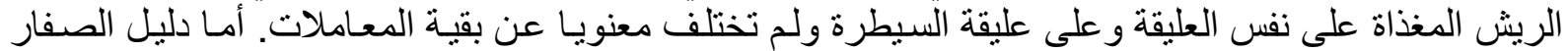

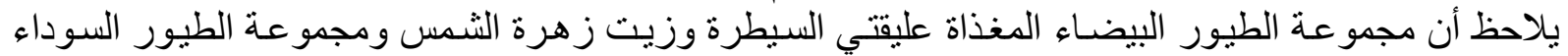

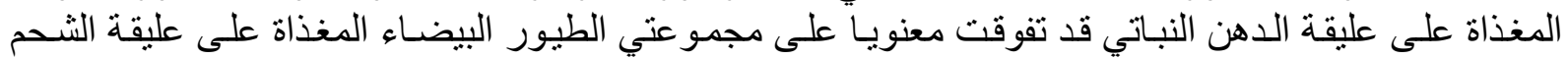

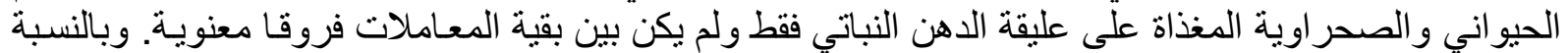

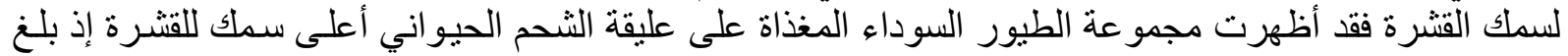


0.42 ملم تفوقت به معنويا على نفس المجموعة من الطيور ولكنها المغذاة على عليقة السيطرة كمـا تفوقت على معلى مجمو عة الطيور الصحر اوية المغذاة على عليقة الدهن النباتي، فيما لم يكن بين بقية المعاملات أي فروق معنوية الطية.

\title{
EFFECT OF GENETIC GROUP AND OIL SOURCE ON THE PERFORMANCE OF LAYING QUAIL
}

\author{
AL-Neemy, M. A. S. \\ Animal Resource Dept., College of Agriculture and Forestry, Mosul University. Iraq \\ E-mail: majid_aln@yahoo.com
}

\begin{abstract}
This study was conducted to evaluate the productive performance (Production period) of three genetic groups different with feather color White, Black and Desert when fed three diets with $3 \%$ from different oil sources compared with control diet $\left(\mathrm{T}_{1}\right)$ namely: sunflower oil $\left(\mathrm{T}_{2}\right)$, vegetable fat $\left(\mathrm{T}_{3}\right)$ and animal grease $\left(\mathrm{T}_{4}\right)$. Two hundred fifty two females, each genetic group 84 females of 7 weeks age were used and distributed randomly to four treatments with 3 replicates per each treatment and every replicate had 7 females. The birds were reared in cages to 12 weeks (the data of first 2 wks. Were as a preliminary period) with ad-libitum feeding. The results revealed that the genetic group with white feather had a significant effect in : average egg number, egg production H.D. , feed consumption, shell with membranes weight and yolk diameter, While the desert birds showed higher live body weight. Also, the oil source had significant effect on the performance as the sunflower oil diet showed a significant effect in: live body weight, average egg number, egg production H.D. , feed consumption, feed conversion and albumen weight. While, there were no significant effect on egg weight. The interaction between genetic group and oil source showed significant effect on all production traits except egg weight.

Keywords: Genetic group, Oil source, Japanese quail.
\end{abstract}

Received: 18/9/2013, Accepted: 2/12/2013.

المصادر

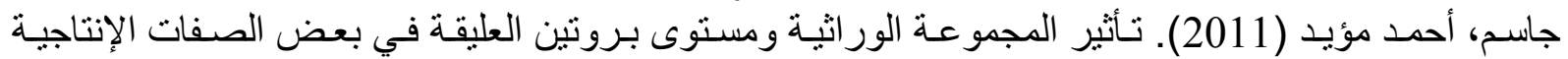
و الدموية لطائر السلوى. رسالة ماجستير / كلية الزراعة والثة الغابات / جامعة الموصل.

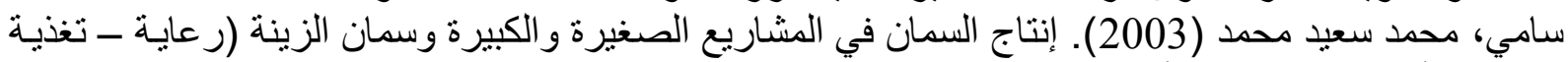

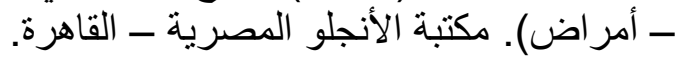

العبيدي، فـارس عبد علي (1999). تقييم الصفات النوعية والكية الكيميائية لبيض طـائر السلوى الياباني. أطروحة

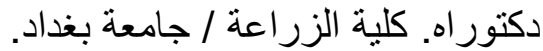

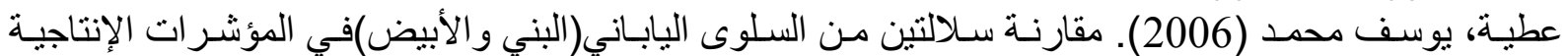

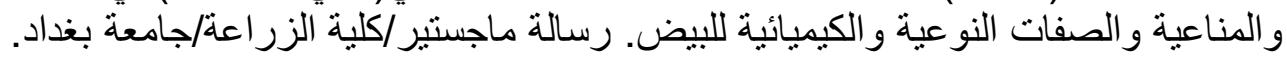

Al-Daraji, H., H.A. Al-Mashadani, H.A.Mirza, W.K.Al-Hayani and A.S. Al-Hassani (2011). Influence of source of oil added to diet on egg quality traits of laying quail. International Journal of Poultry Science 10 (2): 130 - 136.

Al-Daraji, H., H.A. Al-Mashadani, W.K.Al-Hayani, H.A.Mirza and A.S. Al-Hassani (2010). Effect of dietary supplementation with different oils on productive and reproductive performance of quail. International Journal of Poultry Science 9 (5): 429-435. 
Anony mous, (1994). Nutrient Requirements Of Poultry. $9^{\text {th }}$ Rev. Edn., National Academy Press, Washington, DC. U. S. A.

Anony mous, (2000). SAS/STAT User's Guide, Version 6.12.SAS. Inst. Inc., Tekirdag, Turkey, pp: 32-57.

Anthony, N. B, D. A. Emmerson, K. E. Nestor and W. L. Bacon, (1990). Divergent selection for body weight yolk precursor in Coturnix coturnix Japonica 8. A summary of correlated responses. Poultry Science 69: 1055-1063.

Baucells, M., Crespo, N., Barroeta, A.C., Lopez-Ferrer, S. \& Grashorn, M.A., (2000). Incorporation of different polyunsaturated fatty acids into eggs. Poultry science 79: $51-59$.

Guclu, B.K., F.Uyank and K.M. Iscan (2008). Effect of dietary oil sources on egg quality, fatty acid composition of eggs and blood lipids in laying quail. South African Journal Of Animal Science, 38: 91-100.

Midilli, M., I. Bayram, H. Erol, I.S. Cetingul, S. Cakir, E. Calikoglu and M. Kiralan, (2009). The effects of dietary poppy seed oil and sunflower oil on performance, reproduction and egg quality parameters and fatty acid profile of egg yolk in the japanese quail. Journal Animal Vet. Adv., 8: 379-384.

Stadelman. W. J. and O. J. Cotterill., (1998). Egg Science And Technology, A. U. puplishing Co. In Inc. Westport. 
Mesopotamia J. of Agric.

Vol. (45) No. (4) 2017
ISSN: 2224 - 9796 (Online)

ISSN: 1815 - 316 X (Print)
مجلـــــة زر اعـــة الــر افديـنـ

المجلد (45) العدد (4) 2017 